\title{
Wave-based Analysis of Large Nonlinear Photovoltaic Arrays
}

\author{
Alberto Bernardini, Student Member, IEEE, Paolo Maffezzoni, Senior Member, IEEE, \\ Luca Daniel, Member, IEEE and Augusto Sarti, Senior Member, IEEE
}

\begin{abstract}
In this paper, a novel analysis method based on Wave Digital (WD) principles is presented. The method is employed for modeling and efficiently simulating large PhotoVoltaic (PV) arrays under partial shading conditions. The WD method allows rapid exploration of the current-voltage curve at the load of the PV array, given: the irradiation pattern, the nonlinear PV unit model (e.g. exponential junction model with bypass diode) and the corresponding parameters. The Maximum Power Point can therefore easily be deduced. The main features of the proposed method are the use of a scattering matrix that is able to incorporate any PV array topology and the adoption of independent one-dimensional nonlinear solvers to handle the constitutive equations of $P V$ units. It is shown that the WD method can be considered as an iterative relaxation method that always converges to the PV array solution. Rigorous proof of convergence and results about the speed of convergence are provided. Compared to standard Spice-like simulators, the WD method results to be 35 times faster for PV arrays made of some thousands elements. This paves the way to possible implementations of the method in specialized hardware/software for the real time control and optimization of complex PV plants.
\end{abstract}

Index Terms-nonlinear circuits, wave digital filters, PV modeling, scattering, network topology.

\section{INTRODUCTION}

PhotoVoltaic (PV) power systems are among the most promising renewable energy technologies [1], as they are almost pollution-free and particularly suitable for distributed energy generation [2], [3], e.g. through interconnection on the grid [4]. However, it is well known that the actual performance of large PV systems, in terms of generated energy, system reliability and robustness, critically depends on many design factors and working conditions [5]. Very frequently, the energy that is actually harvested by PV plants is smaller than the expected one. This is in part due to the fact that PV plants are complex systems realized by interconnecting, in a modular fashion, a huge number of simple PV units (e.g. solar cells, PV modules or panels) [6]-[8] whose $I-V$ characteristic is nonlinear and critically affected by numerous electrical parameters (e.g. series and parallel resistances [9]) and working conditions (e.g. solar irradiance [10] and local temperature [11]). Furthermore, such working conditions can

A. Bernardini, P. Maffezzoni and A. Sarti are with the Dipartimento di Elettronica, Informazione e Bioingegneria, Politecnico di Milano, Piazza L. Da Vinci 32, 20133 Milano, Italy (e-mail: [alberto.bernardini,paolo.maffezzoni,augusto.sarti]@ polimi.it).

L. Daniel is with Department of Electrical Engineering and Computer Science, Massachusetts Institute of Technology (MIT), 77 Massachusetts Avenue, Cambridge, MA 02139, USA (e-mail:luca@mit.edu). be nonuniform over the PV units giving rise to a great number of different possible operating scenarios that should be predicted and considered during the design phase. It is well known, for instance, that nonuniform solar irradiance, due to partial shading conditions, can heavily deteriorate the system performance with a final impact that indeed depends on how PV units are interconnected [12]. In order to make PV systems more resilient to partial shading conditions, bypass diodes are commonly adopted [13], but this tends to complicate the electrical behavior of the PV systems whose global $I$ $V$ characteristic, at the load of the PV array, can exhibit multiple local maxima [14]. Not only do these facts make an accurate and fast modeling of PV arrays difficult, but they also significantly complicate the design and testing of Maximum Power Point Tracking algorithms [15]-[17].

The efficient exploration of the $I-V$ characteristic of complex PV systems, while considering the many possible scenarios, requires versatile modeling and effective simulation tools that are currently missing. In fact, general purpose simulators, such as Spice-like simulators, reveal to be ineffective when used to simulate large PV systems. This is because such simulators employ the standard Modified Nodal Analysis (MNA) method to formulate the system equations and the multidimensional Newton-Raphson (NR) algorithm as the nonlinear solver. This way, the modular structure of PV systems is not exploited in any way and the nonlinear solver works on all of the equations and unknown variables (i.e. nodal voltages) simultaneously, resulting in slow convergence or even convergence failures. Some simulation techniques specialized for PV systems with particular topologies have been proposed in the literature. For instance in [18], a method for breaking the PV equations into many one-dimensional NR problems has been described; however, its applicability is limited to the simple case where PV units are all connected in series or in parallel. In this paper, instead, an innovative technique is presented for the simulation of large PV systems, which works for whatever type of array topology, and enables the rapid derivation of the $I-V$ characteristic. The presented method relies on Wave Digital (WD) principles [19], as it applies the following transformation to each port of the reference circuit

$$
a=v+Z i \quad b=v-Z i
$$

where $v$ is the port voltage, $i$ is the port current, $Z$ is a real positive free parameter called reference port resistance, while $a$ and $b$ are the so-called incident and reflected wave variables. 
The inverse mapping of (1) is

$$
v=\frac{a+b}{2} \quad i=\frac{a-b}{2 Z} .
$$

Using the simple linear transformation of port variables (1) we are able to separate the constitutive equations of the single PV units (i.e. the $I-V$ characteristic of a solar cell) from the relationships that describe the array interconnection topology. An important contribution of this paper is that of showing how the proposed simulation method corresponds to an iterative relaxation scheme that combines the evaluation of a WD scattering matrix, describing the topology, with a robust and extremely efficient one-dimensional solution of the constitutive equation of PV units. We prove that, for the types of nonlinearities occurring in PV models, the proposed iterative method always converges to the solution. We also show how numerical convergence can be significantly accelerated by dynamically changing some parameters of the WD model.

It is worth noticing that various previous attempts to apply WD method to electrical simulations are available in the literature [20], [21]. Such techniques commonly tend to break the network into small sets of elements coupled through topological junctions called adaptors [22]-[26], and are applied when the network is mainly linear with a few nonlinear elements [27]-[40]. By contrast, our technique has been especially developed for successfully handling the critical case where the majority of elements of the network (at limit all of them) are nonlinear, as it is indeed the case of PV arrays. This ability can be attributed to the use of a topological junction extended to all the elements of the network, as well as by the capability of dynamically changing the free parameters according to the previously found operating point.

The paper is organized as follows. Section II reports some basic concepts about graph representation of circuit network topology. In Section III the scattering matrix embedding the topological information for an arbitrary PV array is derived, while in Section IV the WD equations of the PV array units and their solution by means of the one-dimensional NR algorithm are presented. Section $\mathrm{V}$ describes in detail the novel relaxation technique, referred to as Scattering Iterative Method (SIM), for finding the operating point of the PV array and provides the relative convergence analysis. Section VI presents the overall algorithm for the determination of $I-V$ and $P-V$ curves. Section VII reports some significant numerical results obtained from the application of the algorithm described in Section VI to non-uniformly shaded PV arrays characterized by different topologies and provides efficiency comparisons with Spice-like simulations. Section VIII concludes this paper.

Finally, important implementation details and the proof of the Theorem used for the SIM convergence analysis are described in the Appendix A and Appendix B, respectively. These results, that for the sake of readability are reported in the Appendixes, are among the crucial contributions of this manuscript, both from an implementation point of view and a theoretical perspective.

\section{BACKGROUND}

Let us consider a network composed of $N-1$ nonlinear twoterminal PV units and a load resistor interconnected according to an arbitrary topology; Fig. 1(a) shows an example of the sort. Let us define $\mathbf{v}=\left[v_{1}, \ldots, v_{N}\right]^{t}$ as the vector of port voltages across the elements and $\mathbf{i}=\left[i_{1}, \ldots, i_{N}\right]^{t}$ as the vector of port currents through the elements. The sign conventions of port variables are always chosen as shown in Fig. 1(a) for the PV unit 1 ; the polarity of $v_{1}$ is indicated by the signs + and - , while the polarity of $i_{1}$ is indicated by an arrow. The network topology is commonly described by an oriented graph as shown in Fig. [1(b) [41]. Given the graph of the PV array network, the edges are partitioned in two groups: a set of branches (edges of a tree) and a set of links (edges which are not part of the tree) [41]. Then, according to loop and cut-set analysis, the Kirchhoff laws can be written in vectorial form as

$$
\mathbf{i}=\mathbf{B}^{T} \mathbf{i}_{\mathbf{l}} \quad \mathbf{v}=\mathbf{Q}^{T} \mathbf{v}_{\mathbf{t}}
$$

where $\mathbf{B}$ is the fundamental loop matrix, $\mathbf{Q}$ is the fundamental cut-set matrix and the superscript $T$ denotes transposition [42]. In (3) $\mathbf{v}_{\mathbf{t}}$ is the vector of voltages across branches and $\mathbf{i}_{\mathbf{l}}$ is the vector of currents through links.

Notice that, if $t$ is the number of branches and $l$ is the number of links, $\mathbf{B}$ is a $l \times N$ matrix, while $\mathbf{Q}$ is a $t \times N$ matrix. Moreover, the relation $N=t+l$ holds.

\section{Modeling the Topology}

In this Section, a general method is described that, exploiting the graph representation illustrated in the previous Section [II. allows one to derive a scattering matrix which embeds all the topological information. This will enable modeling the elements of the network (i.e. the PV units and the load resistance) as separated input/output blocks connected to a topological junction characterized by the aforementioned scattering matrix, as shown in Fig. 2

\section{A. Wave Digital Scattering Matrix Derivation}

A linear transformation of the port variables is defined as follows

$$
\mathbf{a}=\mathbf{v}+\mathbf{Z i} \quad \mathbf{b}=\mathbf{v}-\mathbf{Z i},
$$

where $\mathbf{a}=\left[a_{1}, \ldots, a_{N}\right]^{T}$ and $\mathbf{b}=\left[b_{1}, \ldots, b_{N}\right]^{T}$ are vectors of auxiliary variables called incident and reflected waves, respectively, while $\mathbf{Z}=\operatorname{diag}\left[Z_{1}, \ldots, Z_{N}\right]$ is a diagonal matrix, whose non-zero entries are the reference port resistances.

In [43], [44], it is proved that the scattering matrix $\mathbf{S}$ such that

$$
\mathbf{b}=\mathbf{S a}
$$

can be computed using one of the two following (equivalently valid) dual formulas:

$$
\begin{gathered}
\mathbf{S}=2 \mathbf{Q}^{T} \mathbf{K}_{\mathbf{t}}-\mathbf{I}_{N}, \\
\mathbf{S}=\mathbf{I}_{N}-2 \mathbf{K}_{\mathbf{l}} \mathbf{B},
\end{gathered}
$$

where $\mathbf{I}_{N}$ is the $N \times N$ identity matrix, $\mathbf{B}$ and $\mathbf{Q}$ are the fundamental loop matrix and fundamental cut-set matrix, respectively, previously defined in (3) [42]. $\mathbf{K}_{\mathbf{t}}$ is a $t \times N$ matrix such that

$$
\left(\mathbf{Q G Q}^{T}\right) \mathbf{K}_{\mathbf{t}}=\mathbf{Q G}
$$




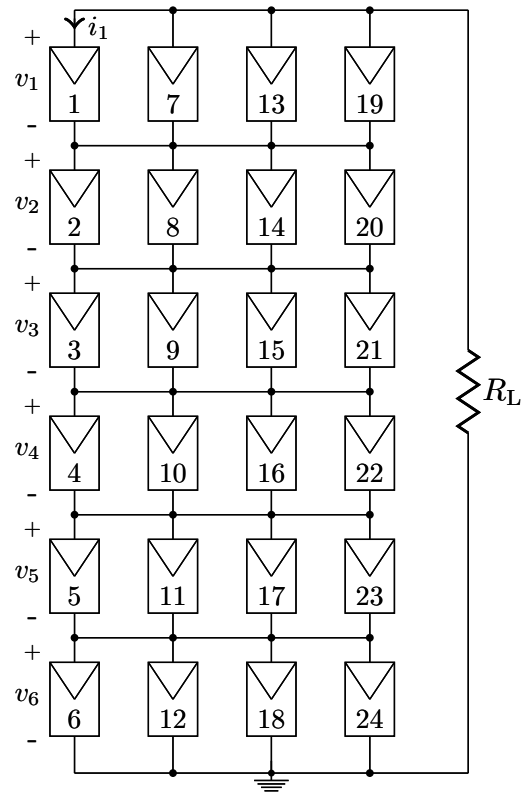

(a) Electrical Circuit

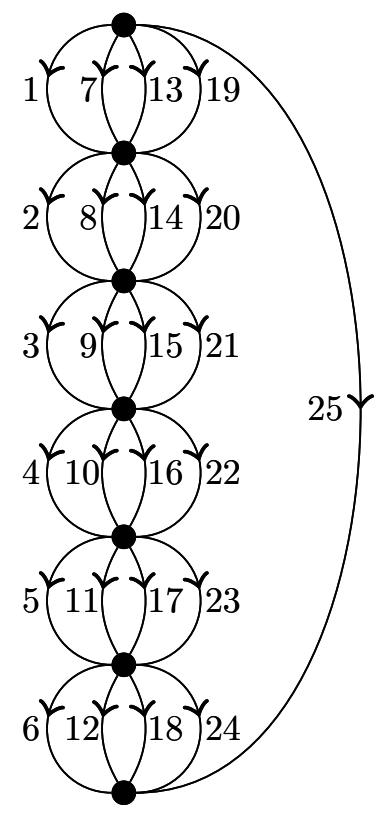

(b) Graph

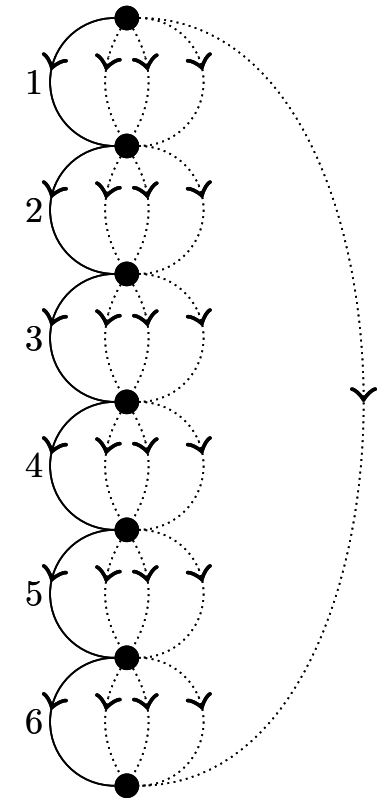

(c) Branches and Links Partition

Fig. 1. Total-Cross-Tied $6 \times 4$ PV Array Topology. In the oriented graph in Fig. 1(c) continuous edges are branches and dotted edges are links.

where $\mathbf{G}=\mathbf{Z}^{-1}$, and $\mathbf{K}_{\mathbf{l}}$ is a $N \times l$ matrix such that

$$
\mathbf{K}_{\mathbf{l}}\left(\mathbf{B Z B}^{T}\right)=\mathbf{Z B}^{T} \text {. }
$$

It follows that for finding $\mathbf{K}_{\mathbf{t}}$ a linear matrix equation in the form (8) with a $t \times t$ coefficient matrix $\mathbf{T}=\mathbf{Q G Q}^{T}$ needs to be solved. Similarly, finding $\mathbf{K}_{\mathbf{l}}$ requires solving a linear matrix equation in the form (9) with a $l \times l$ coefficient matrix $\mathbf{C}=\mathbf{B Z B}^{T}$. It can be verified that the matrices $\mathbf{T}$ and $\mathbf{C}$ are symmetric, positive definite and full rank by construction. Furthermore, in general, the following inequalities hold

$$
\begin{cases}1 \leq \min [t, l] \leq N / 2, & \text { if } N \text { is even } \\ 1 \leq \min [t, l] \leq(N-1) / 2, & \text { if } N \text { is odd }\end{cases}
$$

Consequently, although there are two valid formulas for deriving S, i.e. (6) and (7), one of them is often far cheaper in terms of computational cost. In fact, if $t<l$, (6) is computationally cheaper than (7); conversely, if $l<t$, the opposite holds true. These facts will be clarified by two examples in Subsection III-B

As far as the properties of $\mathbf{S}$ are concerned, it can be proven that $\mathbf{S}$ is a self-inverse (or involutory) matrix [19], [43]-[45], i.e. $\mathbf{S S}=\mathbf{I}_{N}$. As a consequence, (5) can equivalently be written as

$$
\mathbf{a}=\mathbf{S b} \text {. }
$$

It follows that all the eigenvalues of $\mathbf{S}$ have unitary modulus. Moreover, the property $\mathbf{S}^{T} \mathbf{G}=\mathbf{G S}$ holds true; therefore, the $\mathrm{N}$-port scattering junction is said to be reciprocal [45].

\section{B. Examples of Applications: TCT and SP Topologies}

Fig. 1(a) shows a PV array with Total-Cross-Tied (TCT) topology [12]. The corresponding oriented graph is represented in Fig. 1(b) where the orientation of the arrows on the edges

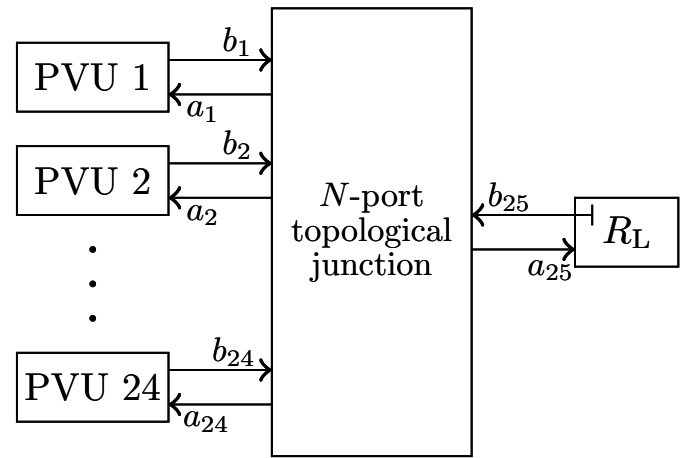

Fig. 2. Wave Digital representation of an arbitrary PV Array. The $N$-port topological junction embeds all the topological information of the PV array, while the elements, i.e. the PV units (PVU) and the load resistance $\left(R_{\mathrm{L}}\right)$, are represented as separated blocks communicating with the junctions through incident and reflected wave signals.

indicates the polarity of the port currents $\mathbf{i}$. The first 24 edges are numbered following the numeration of the PV units and edge 25 corresponds to the load resistance $R_{\mathrm{L}}$. Fig. 1(c)] shows the same graph of Fig. 1(b) and highlights a possible partition of its edges in branches and links; in fact, the subgraph made of continuous edges is a possible tree and the dotted edges are the corresponding links. Note that $t<l$, as $t=6$ and $l=19$; therefore, using (6) is computationally cheaper than using (7) for deriving $\mathbf{S}$. In particular, if $\mathbf{v}_{\mathbf{t}}=\left[v_{1}, \ldots, v_{6}\right]^{T}$ is the vector of port voltages relative to the branches (i.e. the numbered edges in Fig. 1 (c) $]$ and $\mathbf{v}=\left[\mathbf{v}_{\mathbf{t}}{ }^{T}, v_{7}, \ldots, v_{25}\right]^{T}$, the fundamental cut-set matrix $\mathbf{Q}=\mathbf{Q}_{\mathrm{TCT}}$, such that $\mathbf{v}=\mathbf{Q}_{\mathrm{TCT}} \mathbf{v}_{\mathbf{t}}$, can be easily derived by inspection, obtaining:

$$
\mathbf{Q}_{\mathrm{TCT}}=\left[\mathbf{I}_{t}, \mathbf{I}_{t}, \mathbf{I}_{t}, \mathbf{I}_{t}, \mathbb{1}_{t}\right]
$$


where $\mathbf{I}_{t}$ is the $t \times t$ identity matrix and $\mathbb{1}_{t}$ is a $t \times 1$ vector of ones. Let us notice that a generalization of the structure of $\mathrm{Q}_{\mathrm{TCT}}$ for describing a PV array with TCT topology of whichever size is straightforward.

As an alternative example, let us now consider the PV array in Fig. 3(a), characterized by a Series-Parallel (SP) topology. The corresponding oriented graph is represented in Fig. 3(b). Fig. 1(c) shows the same graph of Fig. 1(b) and highlights a possible partition of its edges in branches and links. We notice that $t>l$, as $t=21$ and $l=4$; therefore, this time, using (7) is computationally cheaper than using (6) for deriving $\mathbf{S}$. In particular, if $\mathbf{i}_{\mathbf{l}}=\left[i_{1}, \ldots, i_{4}\right]^{T}$ is the vector of port currents relative to the links (i.e. the numbered edges in Fig. 3(c)p and $\mathbf{i}=\left[\mathbf{i}_{\mathbf{l}}, i_{5} \ldots, i_{25}\right]^{T}$, the fundamental loop matrix $\overline{\mathbf{B}}=$ $\mathbf{B}_{\mathrm{SP}}$, such that $\mathbf{i}=\mathbf{B}_{\mathrm{SP}}^{T} \mathbf{i}_{\mathbf{l}}$, can be easily derived by inspection, obtaining:

$$
\mathbf{B}_{\mathrm{SP}}=\left[\mathbf{I}_{l}, \mathbf{I}_{l}, \mathbf{I}_{l},-\mathbb{1}_{l}\right]
$$

where $\mathbf{I}_{l}$ is the $l \times l$ identity matrix and $\mathbb{1}_{l}$ is a $l \times 1$ vector of ones. Let us notice that a generalization of the structure of $\mathbf{B}_{\mathrm{SP}}$ for describing a PV array with SP topology of whichever size is straightforward.

Plugging (III-B) in (6) and (12) in (7) the scattering matrices can be derived. The scattering matrices also depend on the port resistances contained in matrices $\mathbf{Z}$ and $\mathbf{G}$; in Section VI, we will discuss how to conveniently set such free parameters.

\section{Modeling the Elements}

In this Section, the models of the PV array elements, i.e. load resistance and PV units, are presented. First, the constitutive equations of the elements in terms of Kirchhoff port variables $i$ and $v$ are defined. Second, the corresponding scattering relations involving the WD port variables $a$ and $b$ are deduced.

\section{A. Load Resistance}

The constitutive equation of the load resistance is simply $v=R_{\mathrm{L}} i$ and, applying transformation (2), we obtain the scattering relation

$$
b=\frac{R_{\mathrm{L}}-Z}{R_{\mathrm{L}}+Z} a
$$

Let us notice that, if we set $Z=R_{\mathrm{L}}$, we obtain $b=0$ for whichever value of $a$. The elimination of the dependency of $b$ from $a$ is called adaptation in WDF theory [19] and the resistor $R_{\mathrm{L}}$ is said to be adapted.

\section{B. PV Units}

Various nonlinear models of PV units have been presented in the literature. The single-diode model with series and parallel resistances [46], shown in Fig. 4, is probably the most widespread; therefore, in this manuscript, we decided to focus on it and on an extension, shown in Fig. 5, which also includes a bypass diode. However, the approach presented in this subsection can be used with no restrictions, also for accommodating alternative PV unit models (such as the twodiode model [47] or the multi-exponential junction model [48]).
The PV unit model in Fig. 4 is governed by the following implicit relationship

$f_{\mathrm{J}}(v, i)=I_{s_{\mathrm{J}}}\left(e^{\left(v-R_{\mathrm{S}} i\right) /\left(\eta_{\mathrm{J}} V_{t}\right)}-1\right)+\frac{v-R_{\mathrm{S}} i}{R_{\mathrm{P}}}-i-I_{\mathrm{ph}}=0$

where $v$ is the PV unit voltage, $i$ is the PV unit current, $I_{\mathrm{ph}}$ is the photo-generated current, $I_{s_{\mathrm{J}}}$ is the dark saturation current, $R_{\mathrm{S}}$ is the series resistance, $R_{\mathrm{P}}$ is the parallel resistance, $V_{t}$ is the thermal voltage and $\eta_{\mathrm{J}}$ is the ideality factor. Notice that, if the PV unit is a PV module, $\eta_{\mathrm{J}}$ is proportional to the number of cells in the module connected in series.

As (14) is a transcendental function, finding a scattering relation similar to (13) is not straightforward. A possible approach for finding a closed formula could be using the Lambert Function as discussed in [32], [49]. Another approach would be simply tabulating the nonlinearity or exploiting canonical PWL representations of single-valued functions [50].

However, here an approach based on the NR method is presented. In fact, NR method is known to be very efficient, especially in the one-dimensional case. To this aim, relationships between port variables (1) and (2) are rewritten in the following way [32],

$$
\begin{aligned}
i & =\frac{a-v}{Z}, \\
b & =2 v-a .
\end{aligned}
$$

For a given value $a=a^{(k)}$ of the incident wave at the PV unit, replacing (15) into (14), we are led to a scalar nonlinear equation $h_{\mathrm{J}}(v)=0$ for the $v$ variable. The expressions of $h_{\mathbf{J}}(v)$ and of its derivative with respect to $v, h_{\mathbf{J}}^{\prime}(v)$ are reported in Table I] Such a nonlinear equation can be solved efficiently with the one-dimensional iterative NR algorithm described by the following updating rule

$$
v^{(j)}=v^{(j-1)}-\frac{h_{\mathbf{J}}\left(v^{(j-1)}\right)}{h_{\mathbf{J}}^{\prime}\left(v^{(j-1)}\right)}
$$

where $j \geq 1$ and the superscript between brackets is the iteration index.

NR solver is stopped when the convergence condition $\left|v^{(j)}-v^{(j-1)}\right|<\epsilon$ is satisfied, with $\epsilon$ being a small tolerance (e.g. $\epsilon=10^{-10}$ ). Once convergence condition is met, the port voltage is set to $v=v^{(j)}$ and the reflected wave $b$ is computed by means of 16 .

It is worth noting here how the one-dimensional solver 17 is in general much more robust than multi-dimensional NR solvers used in general-purpose simulators since reasonable bounds on the values that $v$ can assume can be enforced, thus avoiding overflows or divergences.

Finally, the PV unit model can also be extended so as to include a bypass diode as shown in Fig. 5. In this case, the PV unit can be described by using the function [13]

$$
f_{\mathrm{B}}(v, i)=f_{\mathrm{J}}(v, i)-I_{s_{\mathrm{B}}}\left(e^{-v /\left(\eta_{\mathrm{B}} V_{t}\right)}-1\right)=0
$$

where $I_{s_{\mathrm{B}}}$ and $\eta_{\mathrm{B}}$ are the saturation current and the ideality factor of the bypass diode, respectively. Similarly to what has been done for the previous model, the function $h_{\mathrm{B}}(v)$ and its derivative $h_{\mathrm{B}}^{\prime}(v)$ are derived, as shown in Table I, and used by the NR solver. Also in this case, when NR convergence 


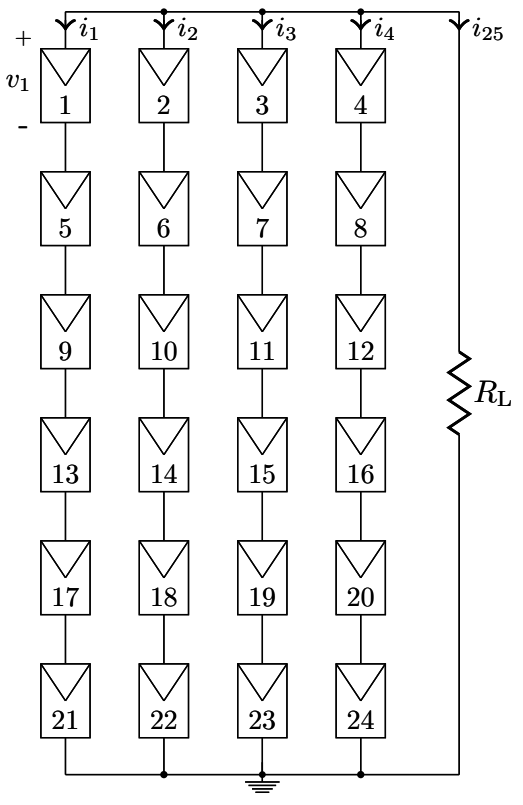

(a) Electric Circuit

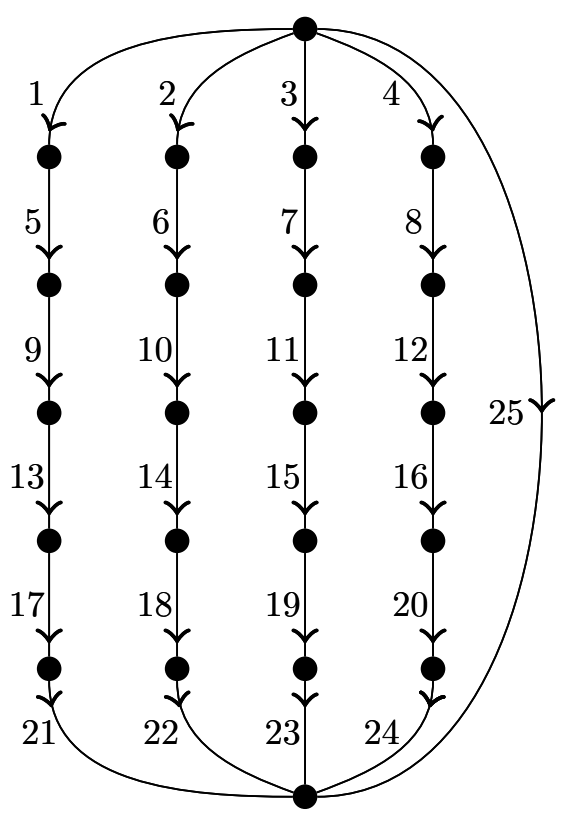

(b) Graph

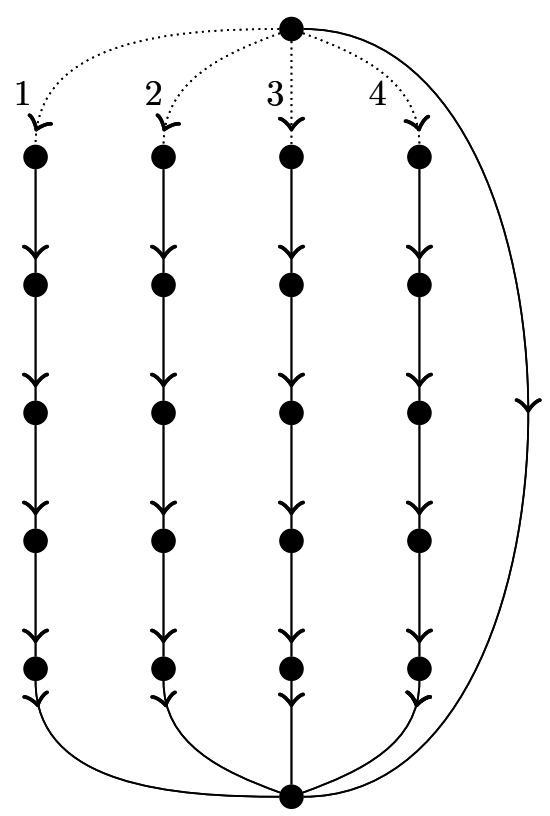

(c) Branches and Links Partition

Fig. 3. Series-Parallel $6 \times 4$ PV Array Topology. In the oriented graph in Fig. 3(c) continuous edges are branches and dotted edges are links.

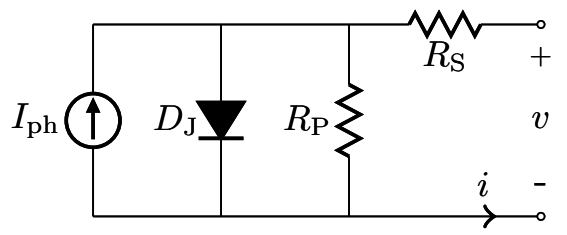

Fig. 4. Single-diode PV unit model with series resistance and parallel resistance.

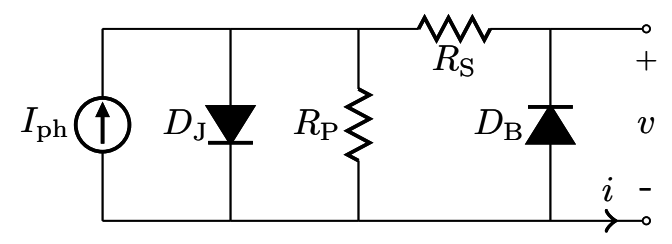

Fig. 5. Single-diode PV unit model with series resistance, parallel resistance and by-pass diode.

condition is satisfied, the reflected wave $b$ is computed using (16).

\section{Computing the Operating Point}

This Section describes the iterative method, later on referred to as Scattering Iterative Method (SIM), for computing the operating point of a PV array, i.e the solution of the PV array for a given value of the load resistance. We will prove that the convergence of SIM, when applied to the proposed PV array models, is always guaranteed while the speed of convergence depends on the free parameters $Z_{1}, \ldots, Z_{N}$.

SIM algorithm is based on the concepts of scattering matrix and one-dimensional PV solvers provided in Section [II] and in Section IV] respectively. Please note that the waves incident to the elements are the waves reflected from the junction and vice versa. In this Section a denotes the vector of waves incident to the elements (or, equally, reflected from the junction), while b denotes the vector of waves reflected from the elements (or, equally, incident to the junction).

\section{A. Scattering Iterative Method (SIM) Description}

The SIM algorithm consists of two main stages, that we will refer to as Local Scattering Stage and Global Scattering Stage. The output of the first Stage is used as the input for the second Stage and vice versa, iteratively, until convergence is achieved. After that, SIM returns the wave vectors $\mathbf{a}$ and $\mathbf{b}$ from which the desired port voltages and port currents can be deduced by means of 21. SIM is implemented as follows.

1) Initialization: vectors $\mathbf{a}$ and $\mathbf{v}$ are set to initial guesses $\mathbf{a}^{(0)}$ and $\mathbf{v}^{(0)}$, respectively (in Section $\mathrm{VI}$ we will discuss how to choose $\mathbf{a}^{(0)}$ and $\mathbf{v}^{(0)}$ ).

2) Local Scattering Stage: the wave reflected from the $n$th element, for $1 \leq n \leq N$, is computed with the onedimensional NR solver and accordingly to 16 . More precisely,

$$
b_{n}^{(k)}=2 v_{n}^{(k)}-a_{n}^{(k-1)}
$$

where $k \geq 1$, the superscript between brackets is the iteration index and $v_{n}^{(k)}$ is the voltage returned by a one-dimensional NR solver, in the cases in which the $n$th element is a PV unit, or simply $v_{n}^{(k)}=a_{n}^{(k-1)} / 2$, in the case in which the $n$th element is the load resistance. The initial guesses of the NR solvers are set as $v_{n}^{(k, j=1)}=v_{n}^{(k-1)}$. Notice that the workload of this stage is embarrassingly parallelizable; in fact, each $b_{n}^{(k)}$ can, in principle, be computed by a separate thread of execution. 
TABLE I

PV UNIT FUNCTIONS USED BY THE NR SOLVERS

\begin{tabular}{|c|}
\hline Single-diode model with series resistance and parallel resistance \\
\hline$h_{\mathrm{J}}(v)=I_{S_{\mathrm{J}}}\left(e^{\left(v\left(Z+R_{\mathrm{S}}\right)-a R_{\mathrm{S}}\right) /\left(\eta_{\mathrm{J}} V_{t} Z\right)}-1\right)+v\left(\frac{R_{\mathrm{S}}+R_{\mathrm{P}}+Z}{R_{\mathrm{P}} Z}\right)-a\left(\frac{1}{Z}+\frac{R_{\mathrm{S}}}{Z R_{\mathrm{P}}}\right)-I_{\mathrm{ph}}$ \\
$h_{\mathrm{J}}^{\prime}(v)=\frac{\partial h_{\mathrm{J}}(v)}{\partial v}=\frac{I_{s_{\mathrm{J}}}\left(R_{\mathrm{S}}+Z\right)}{\eta_{\mathrm{J}} V_{t} Z} e^{\left(v\left(Z+R_{\mathrm{S}}\right)-a R_{\mathrm{S}}\right) /\left(\eta_{\mathrm{J}} V_{t} Z\right)}+\frac{R_{\mathrm{S}}+R_{\mathrm{P}}+Z}{R_{\mathrm{P}} Z}$ \\
\hline Single-diode model with series resistance, parallel resistance and by-pass diode \\
\hline$h_{\mathrm{B}}(v)=h_{\mathrm{J}}(v)-I_{\mathrm{S}_{\mathrm{B}}}\left(e^{-v /\left(\eta_{\mathrm{B}} V_{t}\right)}-1\right)$ \\
$h_{\mathrm{B}}^{\prime}(v)=\frac{\partial h_{\mathrm{B}}(v)}{\partial v}=h_{\mathrm{J}}^{\prime}(v)+\frac{I_{s_{\mathrm{B}}}}{\eta_{B} V_{t}} e^{-v /\left(\eta_{\mathrm{B}} V_{t}\right)}$ \\
\hline
\end{tabular}

3) Global Scattering Stage: being $\mathbf{b}^{(k)}=\left[b_{1}^{(k)}, \ldots, b_{N}^{(k)}\right]^{T}$, the vector $\mathbf{a}^{(k)}=\left[a_{1}^{(k)}, \ldots, a_{N}^{(k)}\right]^{T}$ is evaluated accordingly to

$$
\mathbf{a}^{(k)}=\mathbf{S b}^{(k)} \text {. }
$$

It is worth noticing that: the implementation of formal expression (20) does not need the explicit formation of matrix $\mathbf{S}$ as it is explained in Appendix A

4) Convergence check: convergence is reached when

$$
\frac{\left\|\mathbf{a}^{(k)}-\mathbf{a}^{(k-1)}\right\|_{2}}{\left\|\mathbf{a}^{(k)}\right\|_{2}}<\xi
$$

where the threshold $\xi$ is a small tolerance, e.g. $\xi=10^{-8}$.

\section{B. SIM Convergence Analysis}

In general, the $n$th element (linear or nonlinear) of the PV array is characterized by an implicit relationship between $i_{n}$ and $v_{n}$ in the form

$$
f_{n}\left(v_{n}, i_{n}\right)=0 .
$$

At $k$ th iteration of NR solver, the relationship (22) is linearized around the current $i_{n}^{(k)}, v_{n}^{(k)}$ values. Such a linearization corresponds to a Thévenin equivalent circuit with equation

$$
v_{n}^{(k)}-V_{\mathrm{G} n}^{(k)}-R_{\mathrm{G} n}^{(k)} i_{n}^{(k)}=0,
$$

where $V_{\mathrm{G} n}^{(k)}$ denotes the equivalent source and $R_{\mathrm{G} n}^{(k)}$ the series resistance. Fig. 6 shows the NR linearization of the PV characteristic and the resulting Thévenin equivalent circuit. In particular, the series resistance $R_{\mathrm{G} n}^{(k)}$ can be defined as the implicit derivative of $f_{n}\left(v_{n}, i_{n}\right)$ with respect to $i_{n}$ evaluated at the point with coordinates $v_{n}^{(k)}$ and $i_{n}^{(k)}$

$$
R_{\mathrm{G} n}^{(k)}=\frac{d}{d i_{n}}\left[f_{n}\left(v_{n}^{(k)}, i_{n}^{(k)}\right)\right]=-\frac{\partial f_{n}\left(v_{n}^{(k)}, i_{n}^{(k)}\right) / \partial i_{n}}{\partial f_{n}\left(v_{n}^{(k)}, i_{n}^{(k)}\right) / \partial v_{n}} .
$$

It follows that the equivalent voltage source can be expressed as $V_{\mathrm{G} n}^{(k)}=v_{n}^{(k)}-R_{\mathrm{G} n}^{(k)} i_{n}^{(k)}$. According to 19, we also have that

$$
v_{n}^{(k)}=\frac{a_{n}^{(k-1)}+b_{n}^{(k)}}{2} \quad, \quad i_{n}^{(k)}=\frac{a_{n}^{(k-1)}-b_{n}^{(k)}}{2 Z_{n}} .
$$

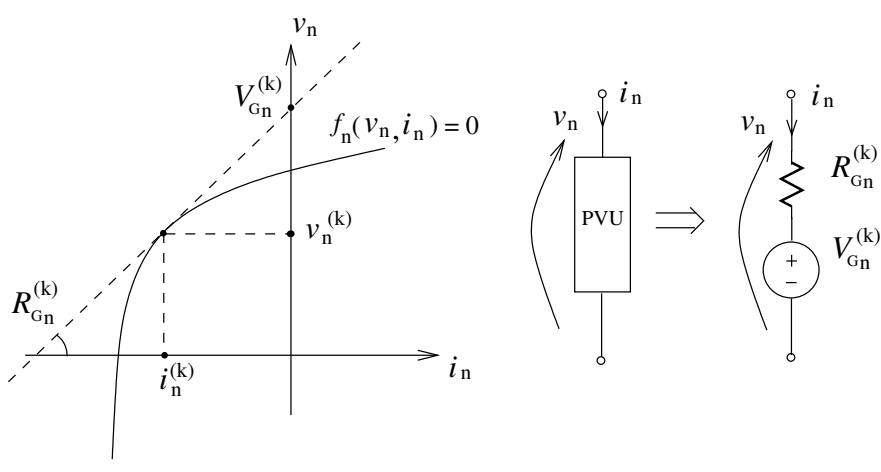

Fig. 6. NR linearization of the PV characteristic at operating point $\left(i_{n}^{(k)}, v_{n}^{(k)}\right)$ and the resulting Thévenin equivalent circuit.

The linearized constitutive equations (23) can be recast in terms of waves variables by substituting (25) into (23). In matrix form, the linearized WD scattering relations result

$$
\mathbf{b}^{(k)}=\mathbf{D}^{(k)} \mathbf{a}^{(k-1)}+\mathbf{E}^{(k)} \mathbf{V}_{\mathrm{G}}{ }^{(k)},
$$

where $\mathbf{D}^{(k)}=\operatorname{diag}\left[c_{1}^{(k)}, \ldots, c_{N}^{(k)}\right]^{T}$ is a diagonal matrix, whose nonzero entries are the reflection coefficients

$$
c_{n}^{(k)}=\frac{R_{\mathrm{G} n}^{(k)}-Z_{n}}{R_{\mathrm{G} n}^{(k)}+Z_{n}},
$$

$\mathbf{E}^{(k)}=\operatorname{diag}\left[e_{1}^{(k)}, \ldots, e_{N}^{(k)}\right]^{T}$ is a diagonal matrix, whose nonzero entries are the weights

$$
e_{n}^{(k)}=\frac{2 Z_{n}}{R_{\mathrm{G} n}^{(k)}+Z_{n}},
$$

and $\mathbf{V}_{\mathrm{G}}{ }^{(k)}=\left[V_{\mathrm{G} 1}^{(k)}, \ldots, V_{\mathrm{G} N}^{(k)}\right]^{T}$. Then, combining (26) and (20), we obtain

$$
\mathbf{a}^{(k)}=\mathbf{S D}^{(k)} \mathbf{a}^{(k-1)}+\mathbf{S E}^{(k)} \mathbf{V}_{\mathrm{G}}^{(k)},
$$

which shows how the SIM algorithm, combined with the local linearization of NR solver, results in an iterative solver having iteration matrices $\mathbf{S D}^{(k)}$. A sufficient condition for SIM to converge is thus given by

$$
\rho\left(\prod_{k=1}^{K} \mathbf{S D}^{(k)}\right)<1
$$


, for any index $K \geq 1$, where

$$
\rho\left(\prod_{k=1}^{K} \mathbf{S D}^{(k)}\right)=\max \left\{\left|\lambda_{1}\right|, \ldots,\left|\lambda_{N}\right|\right\}
$$

is the spectral radius of matrix $\prod_{k=1}^{K} \mathbf{S D}^{(k)}$ and $\left|\lambda_{n}\right|$ with $1 \leq n \leq N$ is the absolute value of the $n$th eigenvalue of the same matrix $\prod_{k=1}^{K} \mathbf{S D}^{(k)}$.

In Appendix $B$, we prove the following theorem:

Theorem 5.1: Let $\mathbf{S}$ be a $N \times N$ scattering matrix based on the voltage wave definition (4), and let $\left\{\mathbf{D}^{(1)}, \ldots, \mathbf{D}^{(K)}\right\}$ be a set of $K \geq 1$ diagonal matrices with dimensions $N \times N$. The following inequality always holds true

$$
\rho\left(\prod_{k=1}^{K} \mathbf{S D}^{(k)}\right) \leq \prod_{k=1}^{K} \rho\left(\mathbf{D}^{(k)}\right)
$$

where $\rho\left(\prod_{k=1}^{K} \mathbf{S D}^{(k)}\right)$ and $\rho\left(\mathbf{D}^{(k)}\right)$ are the spectral radii of $\prod_{k=1}^{K} \mathbf{S D}^{(k)}$ and $\mathbf{D}^{(k)}$, respectively.

According to 31], if

$$
\max \left\{\left|c_{1}^{(k)}\right|, \ldots,\left|c_{N}^{(k)}\right|\right\}<1
$$

for each $k$, convergence of SIM is guaranteed, as $\rho\left(\mathbf{D}^{(k)}\right)=$ $\max \left\{\left|c_{1}^{(k)}\right|, \ldots,\left|c_{N}^{(k)}\right|\right\}$ and the condition $\prod_{k=1}^{K} \rho\left(\mathbf{D}^{(k)}\right)<1$ surely holds true. Finally, remembering that the free parameters $Z_{n}$, for any $n$, are selected to be positive, $Z_{n}>0$, 32 reduces to

$$
R_{\mathrm{G} n}^{(k)}>0
$$

where $R_{\mathrm{G} n}^{(k)}$ are defined in 24.

In the light of the above, the convergence proof of SIM algorithm when applied to PV arrays, reduces to prove that the implicit derivative of $\mathrm{PV}$ unit constitutive equation $f(v, i)$ w.r.t. $i$ is always positive for each element.

In the case of the load resistance, the derivative of $f(v, i)=$ $R_{\mathrm{L}} i-v$ w.r.t. $i$ is always positive and equal to $R_{\mathrm{L}}$. Let us now consider $f(v, i)=f_{\mathrm{J}}(v, i)$; in this case, we have

$$
\frac{d}{d i}\left[f_{\mathrm{J}}(v, i)\right]=\frac{1+R_{\mathrm{S}} / R_{\mathrm{P}}+\left(I_{s_{\mathrm{J}}} R_{\mathrm{S}} /\left(\eta_{\mathrm{J}} V_{t}\right)\right) e^{\left(v-R_{\mathrm{S}} i\right) /\left(\eta_{\mathrm{J}} V_{t}\right)}}{1 / R_{\mathrm{P}}+\left(I_{s_{\mathrm{J}}} /\left(\eta_{\mathrm{J}} V_{t}\right)\right) e^{\left(v-R_{\mathrm{S}} i\right) /\left(\eta_{\mathrm{J}} V_{t}\right)}}
$$

which is always positive since PV unit physical parameters are positive. Let us then consider $f(v, i)=f_{\mathrm{B}}(v, i)$; in this case we have

$$
\frac{d}{d i}\left[f_{\mathrm{B}}(v, i)\right]=\frac{-\partial f_{\mathrm{J}}(v, i) / \partial i}{\partial f_{\mathrm{J}}(v, i) / \partial v+\left(I_{s_{\mathrm{B}}} /\left(\eta_{\mathrm{B}} V_{t}\right)\right) e^{-v /\left(\eta_{\mathrm{B}} V_{t}\right)}}
$$

which, again, is always positive.

\section{SIM Convergence Speed}

It has been shown that SIM converges for any initial guess; however, its convergence speed depends on the magnitude of the nonzero entries of $\mathbf{D}^{(k)}$ (30). Let us notice that the closer $Z_{n}$ is to $R_{\mathrm{Gn}}^{(k)}$, the smaller is the spectral radius of $\mathbf{S D}^{(k)}$ and the faster is SIM, up to the ideal case of full adaptation in which $Z_{n}=R_{\mathrm{G} n}^{(k)}$ holds at each port $n$, the spectral radius of $\mathbf{S D}^{(k)}$ reduces to zero and only one iteration is needed.

\section{COMPuting The LOAD I-V AND P-V CHARACTERISTICS}

In this Section, more details about Algorithm 1 are provided. The algorithm enables efficient computation of the load current-voltage and power-voltage characteristics for large PV arrays under partial shading conditions. The method works for whichever type of PV array configuration, PV model parameters and for arbitrary shading patterns. The algorithm is based on the application of the iterative method, SIM, described in Section $\nabla$ over a wide set of different load resistance values. Such values are decided by sweeping in a logarithmic fashion a range of load which goes from the highest chosen value, e.g. $R_{\mathrm{L} 1}=10^{3} \Omega$, to the lowest one, e.g. $R_{\mathrm{L} M}=10^{-3} \Omega$, and that are ordered in the vector $\mathbf{R}_{\mathrm{L}}=\left[R_{\mathrm{L} 1}, \ldots, R_{\mathrm{L} M}\right]$, where $M$ is the total number of chosen values. The SIM algorithm computes all of the currents and voltages in the PV array for each load resistance in $\mathbf{R}_{\mathrm{L}}$. The currents and voltages at the load resistance are organized in the vectors $\mathbf{i}_{\mathrm{L}}=\left[i_{\mathrm{L} 1}, \ldots, i_{\mathrm{L} M}\right]$ and $\mathbf{v}_{\mathrm{L}}=\left[v_{\mathrm{L} 1}, \ldots, v_{\mathrm{L} M}\right]$, respectively, and determine the operating points over the load $I-V$ characteristic. Similarly, the vector of the load powers is easily derived as $\mathbf{w}_{\mathrm{L}}=\left[w_{\mathrm{L} 1}, \ldots, w_{\mathrm{L} M}\right]=\left[i_{\mathrm{L} 1} v_{\mathrm{L} 1}, \ldots, i_{\mathrm{L} M} v_{\mathrm{L} M}\right]$.

As the first step, all of the PV units parameters, e.g. the assumed irradiance levels and patterns, temperature, ideality factors and shunt or series resistances are set. Then, the port resistances, which are free parameters, of the PV units are initialized to admissible slope values of the PV implicit relationship $f_{n}\left(v_{n}, i_{n}\right)=0$, e.g. values $Z_{n} \in(0.1,1) \Omega$ can be selected for $n=1, \ldots N-1$, while the port resistance of the load is adapted, i.e. $Z_{N}=R_{\mathrm{L} 1}$, as explained in Subsection IV-A

The unknown vectors of incident waves and port voltages are initialized to arbitrary low values, e.g. $a_{n}^{(0)}=0$ and $v_{n}^{(0)}=0.2$. Hence, for each load resistance in $\mathbf{R}_{\mathrm{L}}$, the $\mathrm{PV}$ array is solved iteratively by means of a sequence of Local Scattering Stage and Global Scattering Stage evaluations explained in Subsection $\mathrm{V}-\mathrm{A}$. These stages consist in onedimensional NR solvers, i.e. SOLVE routine in Algorithm 1 and scattering matrix evaluation, accordingly to the efficient procedure described in Appendix A Once SIM has reached convergence, the implicit derivatives 34 or 35) (depending on the used PV unit models), evaluated at the current operating point are employed to set the free parameters $Z_{n}$ to be used at subsequent operating point solution. Similarly, the port variable values $\mathbf{a}$ and $\mathbf{v}$, determined at the current operating point, are used as initial values $\mathbf{a}^{(0)}$ and $\mathbf{v}^{(0)}$ for the NR solver at the next operating point. Since, the Thévenin equivalents of the PV units at two successive operating points on the current-voltage curve are expected to be very close, the above initialization and dynamic change of the free parameters dramatically increases SIM convergence speed, as highlighted in Subsection V-C

\section{NUMERICAL RESULTS}

In this Section, some application examples of the algorithm presented in Section VI are illustrated. In the first example, the proposed WD algorithm is employed to efficiently calculate the load characteristics of PV arrays formed by 24 units, 


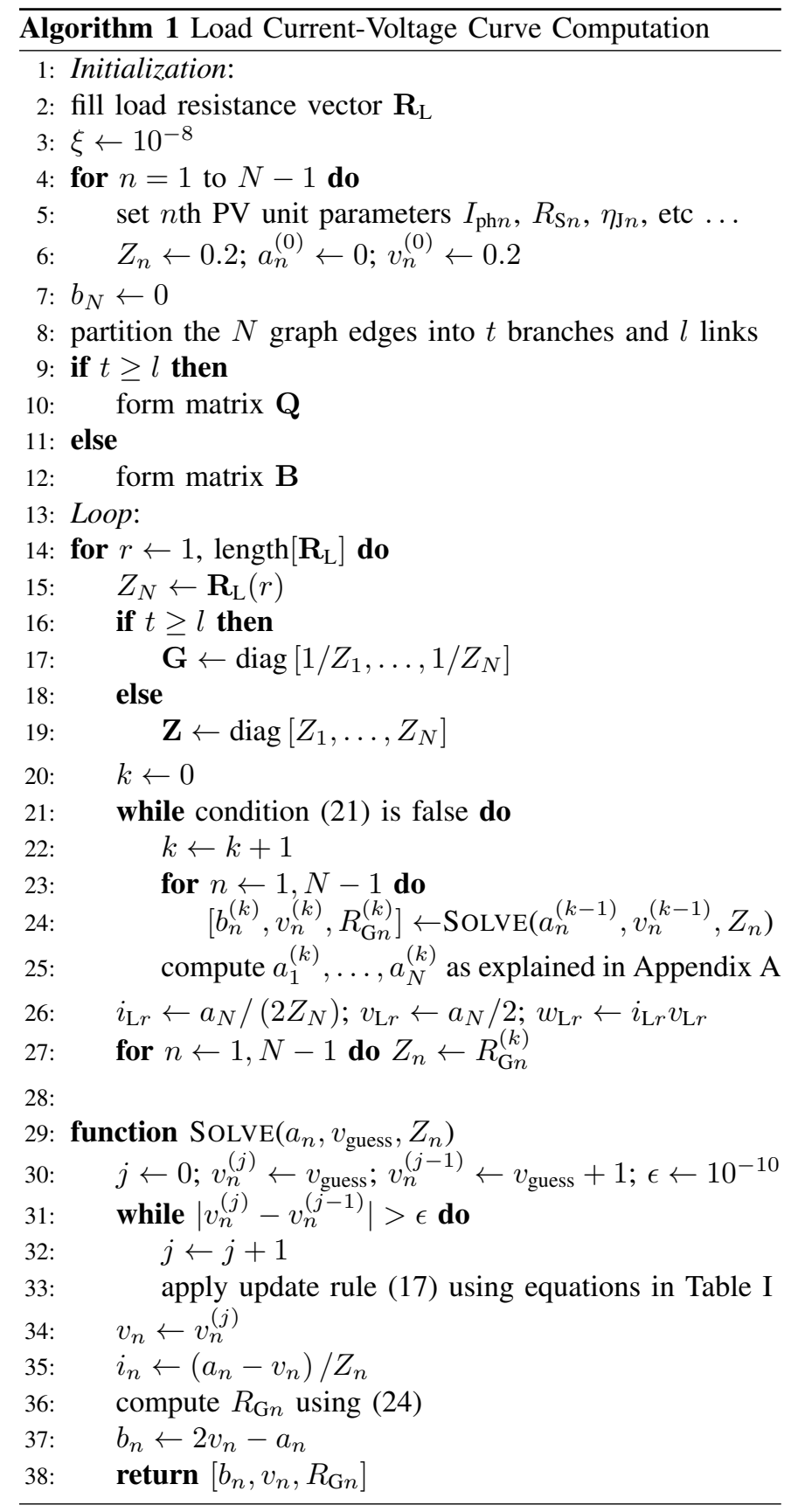

interconnected accordingly to four different topologies widely used in practical applications and working under partial shading condition. This first example shows how the WD method works for arbitrary array topologies, providing simulation results that match accurately with those obtained via Spice-like simulations but with a $7 \times$ simulation time speed-up factor. As the Spice-like simulator we adopt Cadence Spectre [51]. The second example, is used to compare the computational time required by the WD method with that of Spectre simulations when the size of the PV arrays is increased.

\section{A. Analysis of different PV Array Topologies}

In this first example, we consider arrays made of $6 \times 4$ PV units having the four topologies shown in Figs. 1(a), 3(a), 7 and 8 The formation of fundamental loop and

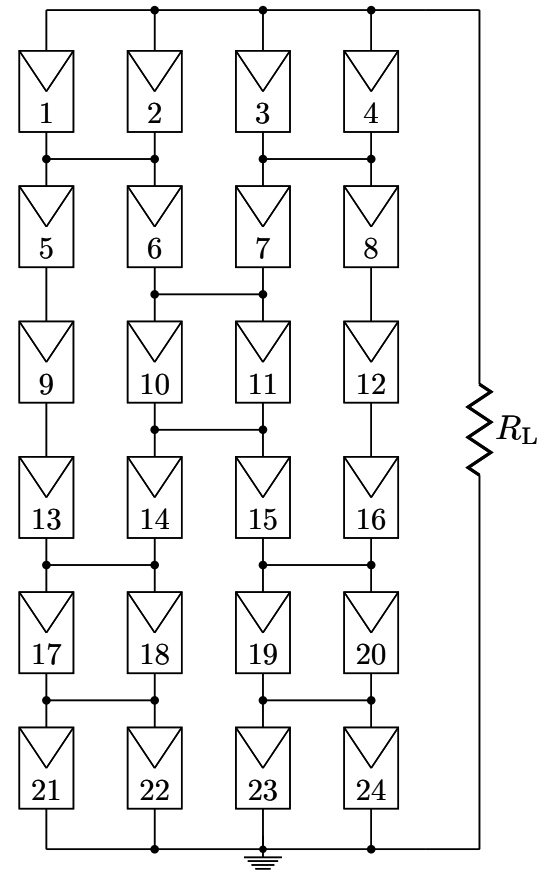

Fig. 7. Bridge-Link $6 \times 4$ PV Array Topology.

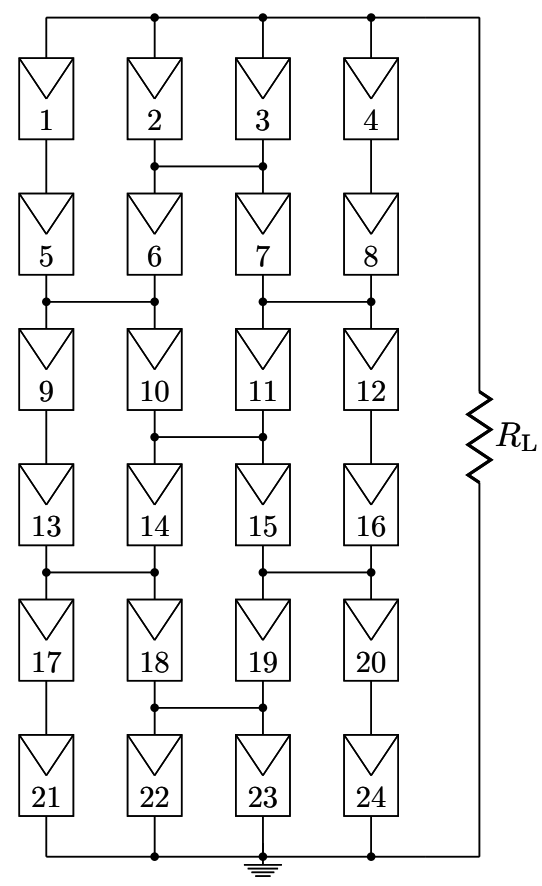

Fig. 8. Honey-Comb $6 \times 4$ PV Array Topology.

cut-set matrix for the TCT and SP topologies in Figs. 1(a) and 3(a) have already being explained in Subsection III-B The array topologies shown in Figs. 7 and 8 instead are characterized by the so called Bridge-Link (BL) and HoneyComb (HC) topologies, respectively. Even though these latter two topologies are more complex than the ones described in Subsection III-B, as they are no more simple combinations of parallel and/or series interconnections, the process for deriving their fundamental loop or cut-set matrix remains the same. In 


\begin{tabular}{|c|c|c|c|}
\hline 0.1 & 1 & 2 & 0.5 \\
\hline 1 & 0.1 & 0.1 & 1 \\
\hline 1 & 1 & 2 & 1 \\
\hline 2 & 2 & 2 & 2 \\
\hline 1 & 1 & 2 & 2 \\
\hline 1 & 0.1 & 0.1 & 0.1 \\
\hline
\end{tabular}

(a) Shading Pattern 1

\begin{tabular}{|c|c|c|c|}
\hline 0.001 & 0.001 & 1 & 1 \\
\hline 0.001 & 1 & 1 & 1 \\
\hline 1 & 1 & 1 & 1 \\
\hline 1 & 1 & 1 & 1 \\
\hline 1 & 1 & 1 & 1 \\
\hline 1 & 1 & 1 & 1 \\
\hline
\end{tabular}

(b) Shading Pattern 2
Fig. 9. Examples of possible shading patterns for $6 \times 4 \mathrm{PV}$ arrays as the ones in Fig. 1(a) 3(a) 7 and 8 Each entry of the shading pattern matrices contains the value of irradiance in ampere for the PV unit placed at the corresponding position.

particular the edges of the graph associated to the BL topology in Fig. 7 are partitioned into 12 branches and 13 links, while the edges of the graph associated to the HC topology in Fig. 7 are partitioned into 13 branches and 12 links.

The models of the PV units is that shown in Fig. 5 with the following parameter values: $V_{t}=26 \times 10^{-3} \mathrm{~V}, I_{s}=10^{-10}$ $\mathrm{A}, I_{s_{\mathrm{B}}}=10^{-11} \mathrm{~A}, \eta_{\mathrm{J}}=1, \eta_{\mathrm{B}}=1, R_{\mathrm{S}}=0.0043 \Omega$ and $R_{\mathrm{P}}=140 \Omega$.

Then, the partial shading pattern shown in Fig. 9(a) is imposed on the arrays, where the entries of the shading pattern matrix are the values of the photo-generated currents $I_{\mathrm{ph} n}$ (measured in ampere) in the $n$th PV unit.

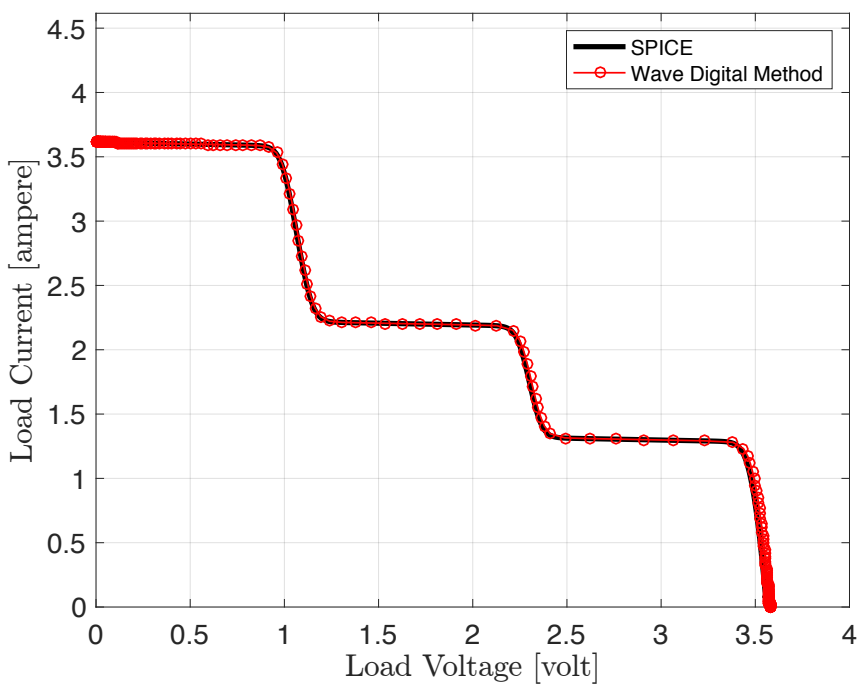

Fig. 10. Load Current-Voltage curve of the PV array with TCT topology in Fig. 1(a) shaded by the shading pattern in Fig. 9(a)

Figs. 10, 12, 14 and 16 reported the profiles of the load current-voltage curves for the four PV arrays with TCT, SP, $\mathrm{BL}$ and $\mathrm{HC}$ topologies, respectively, as computed by the WD method and Spice-like simulation. Similarly, Figs. 11, 13,15 and 17 show the corresponding power-current curves.

It can be seen how the results of the WD method match very well those of the Spice-like simulation. In fact, the difference between the curves provided by the two methods are less than $10^{-6}$ ampere for currents and $10^{-6}$ watt for

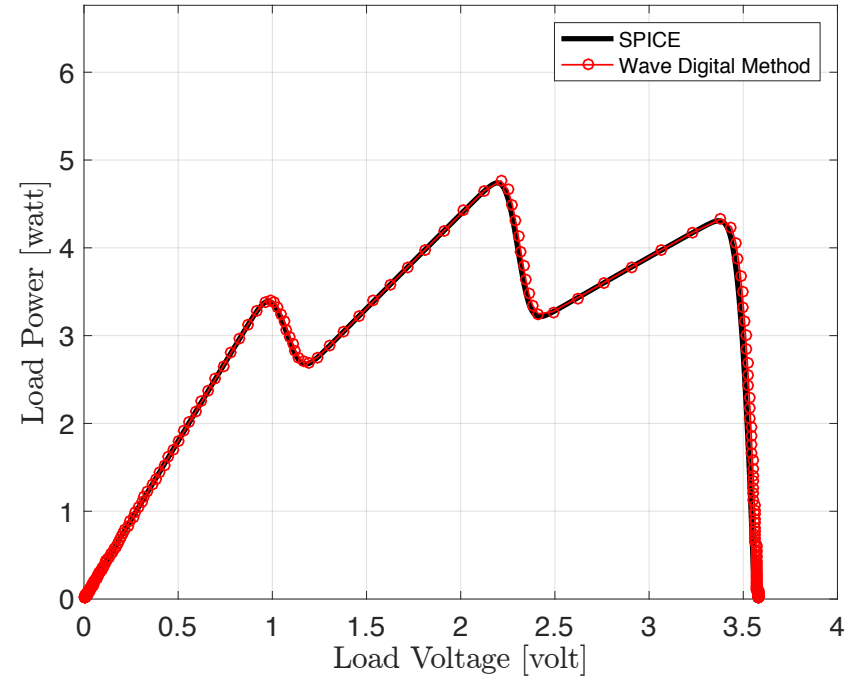

Fig. 11. Load Power-Voltage curve of the PV array with TCT topology in Fig. 1(a) shaded by the shading pattern in Fig. 9(a)

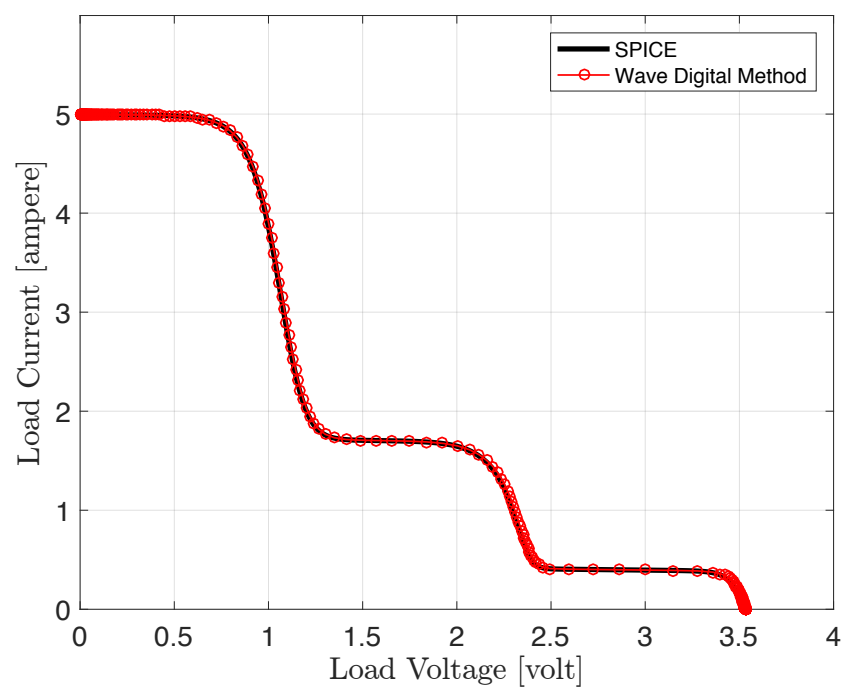

Fig. 12. Load Current-Voltage curve of the PV array with SP topology in Fig. 3(a) shaded by the shading pattern in Fig. 9(a)

powers. In the presence of partial shading, the load $P-V$ characteristic exhibits a complex shape with many hills and valleys thus requiring a great number of simulation points for a reliable description. The proposed WD method allows detailed exploration of such a characteristic in very short simulation times (i.e. less than $0.1 \mathrm{~s}$ for the considered arrays simulated on a standard quad-core computer). In the light of the above considerations, the described WD algorithm can be used in real time applications for finding the load condition that tracks the Maximum Power Point or to identify the best interconnection topology in the case of reconfigurable PV arrays.

Comparing the power curves in Figs. 11, 13 and 15, it is evident how the TCT topology outperforms SP and BL topologies both in terms of maximum supplied power and load voltage figures of merit. From Figs. 11 and 17, instead, it is seen that TCT and HC topologies give comparable performance with TCT exhibiting a slightly higher maximum 


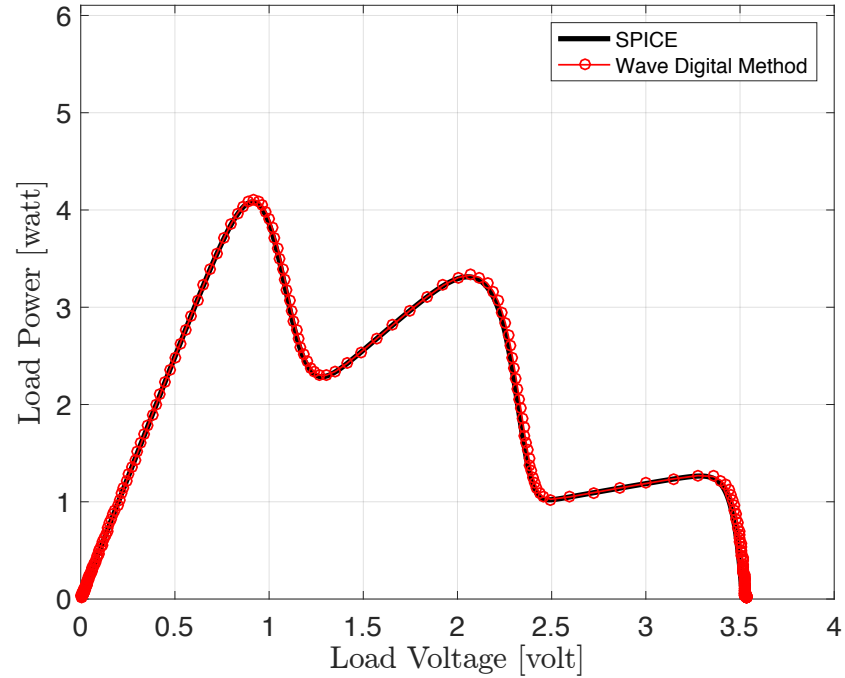

Fig. 13. Load Power-Voltage curve of the PV array with SP topology in Fig. 3(a) shaded by the shading pattern in Fig. 9(a)

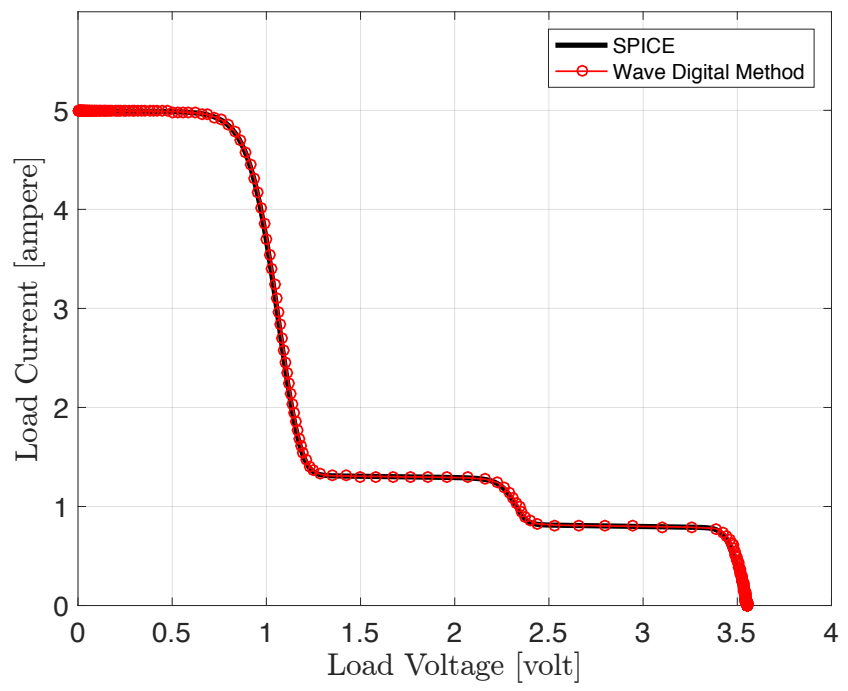

Fig. 14. Load Current-Voltage curve of the PV array with BL topology in Fig. 7 shaded by the shading pattern in Fig. 9(a)

power. As a further quantitative investigation, we exploit the WD method in connection with Monte Carlo simulation to perform variability analysis of the maximum supplied power by considering a large number of possible shading patterns. At each Monte Carlo realization, a randomly selected number of PV units, ranging from 1 to 10 , and with randomly selected positions within the array are fully shaded and $P-V$ characteristic recomputed. Fig. 18 reports the distributions (for 1000 realizations) of the maximum power supplied by TCT, $\mathrm{HC}, \mathrm{BL}$ and SP topologies. The mean values of the maximum supplied power are: $6.9,5.9,5.9$ and 5.2 watt for TCT, HC, $\mathrm{BL}$ and SP, respectively.

\section{B. Comparison to Spice-like Software Performance}

In this second example, the performance of WD algorithm is compared to that of MNA-based Spice-like codes when

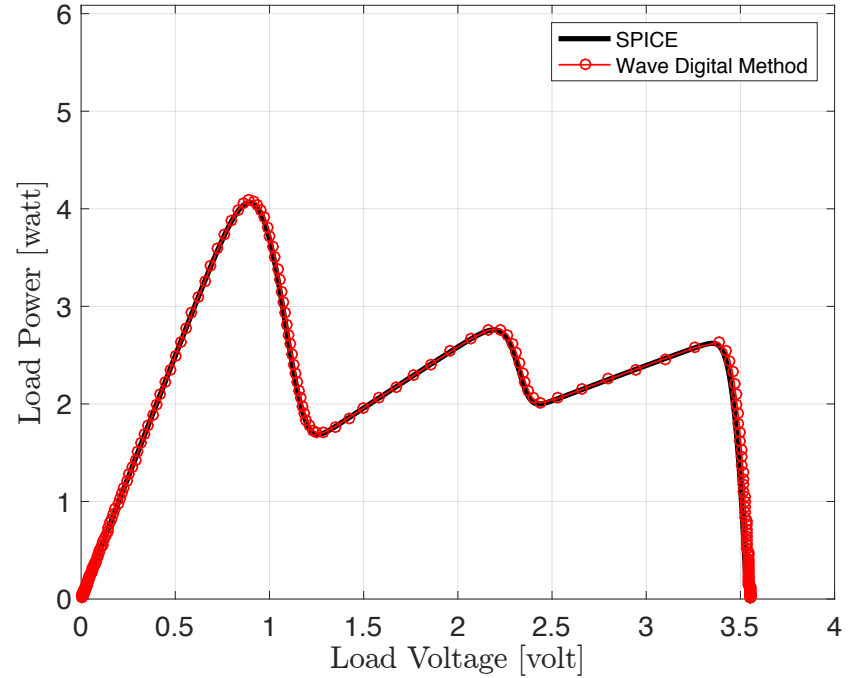

Fig. 15. Load Power-Voltage curve of the PV array with BL topology in Fig. 7 shaded by the shading pattern in Fig. 9(a)

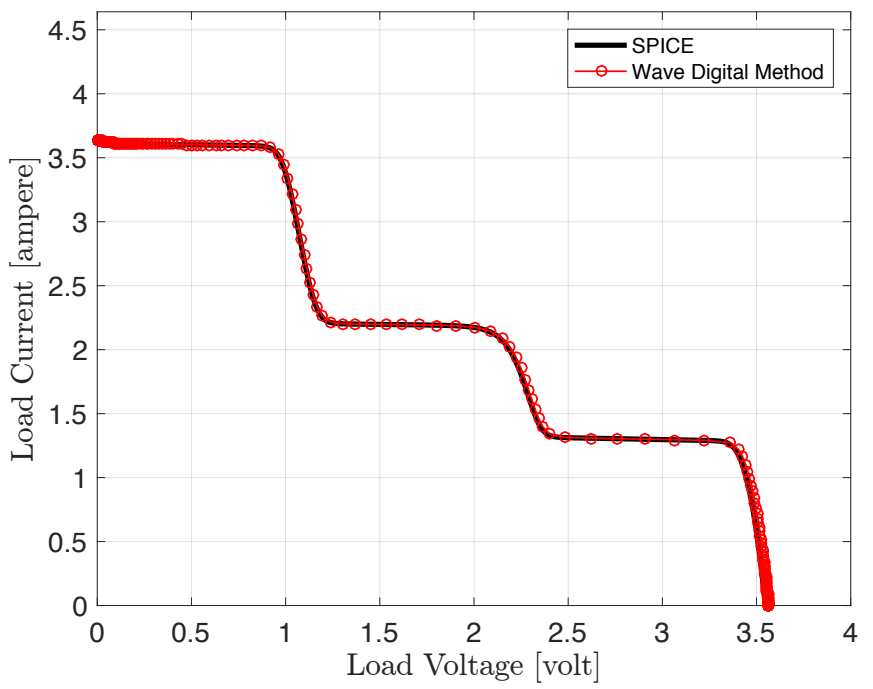

Fig. 16. Load Current-Voltage curve of the PV array with HC topology in Fig. 8 shaded by the shading pattern in Fig. 9(a)

simulating PV arrays of large size. In doing that, we focus on TCT topology with growing number of PV units.

The parameters of the PV units are the same provided in Subsection VII-A. The PV arrays are assumed to be subjected to scaled versions of the shading pattern shown in Fig. 9(b) Table III reports the computation times for WD algorithm and Spice-like simulations performed on the same quad-core processor. Is is apparent how the WD method outperforms the MNA-based method and its benefit in terms of efficiency becomes more pronounced for larger PV arrays. For the case with $6000 \mathrm{PV}$ units, the WD method results $\approx 33 \times$ faster than Spice-like simulation. The computational efficiency of WD method over Spice-like simulations is mainly due to the smaller number and smaller size of the linear systems that the WD method requires solving. With MNA used in Spice-like simulation, the solution at each operating point needs several iterations of the Newton-Raphson algorithm with each iteration 


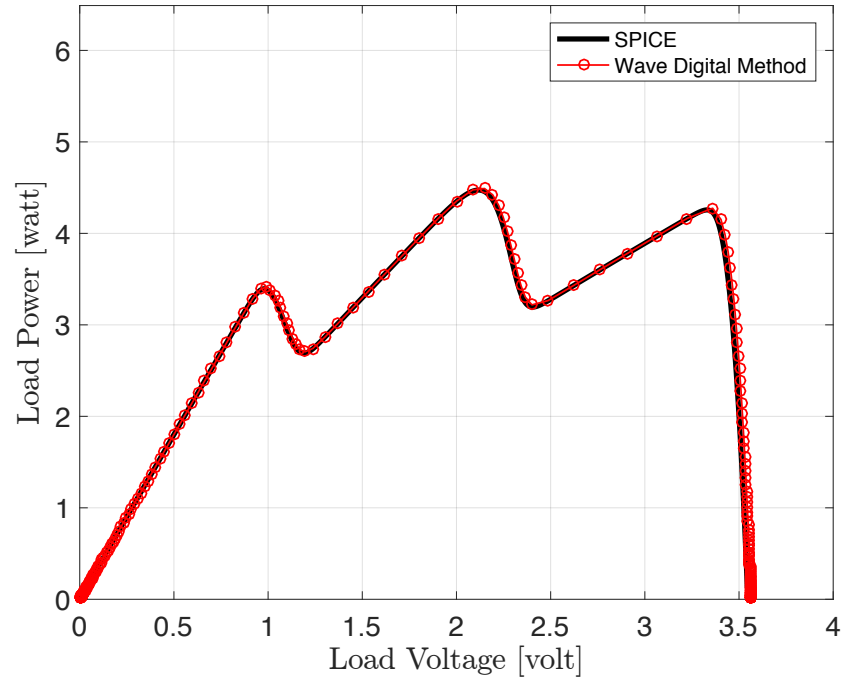

Fig. 17. Load Power-Voltage curve of the PV array with HC topology in Fig. 8 shaded by the shading pattern in Fig. $9(\mathrm{a})$
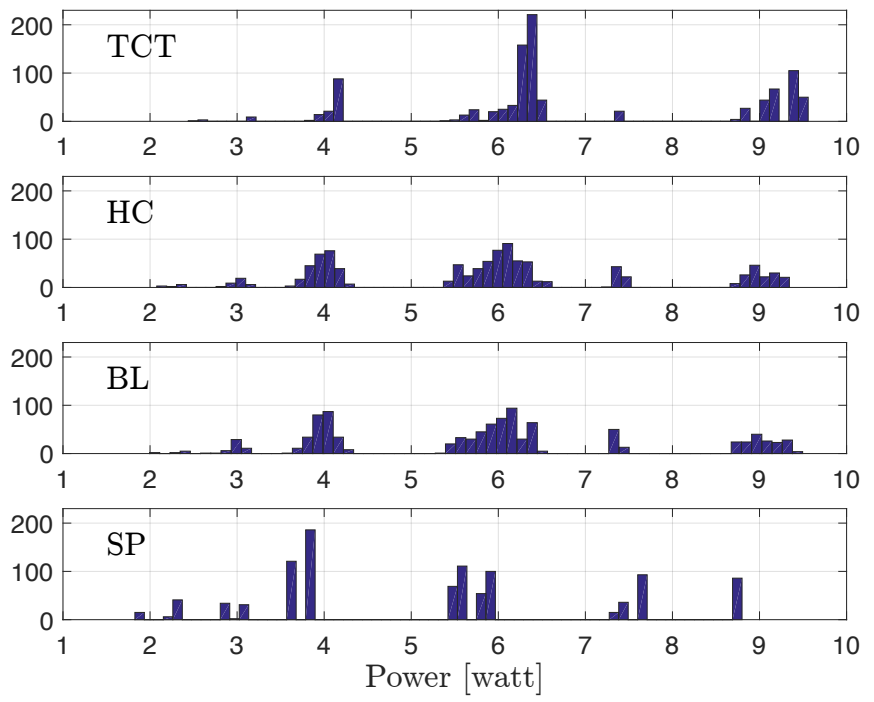

Fig. 18. Maximum power distributions (number of realizations versus maximum power) for TCT, HC, BL and SP topological configurations.

requiring the solution of a linear system. If $N$ denotes the number of PV units, the linear systems of MNA have size $\approx 3 \times N$ (i.e. the number of nodes). By contrast, according to the efficient implementation described in the Appendix A the WD method, at each operating point, requires solving a single linear system. The size of such systems is always $\leq N / 2$. When $N$ gets large, the linear system solution dominates the computational task thus resulting in the simulation speed up shown in Fig. 19

Another important issue when dealing with large PV systems is simulation robustness. In Spice-like simulations of large arrays with $N>2000$ we were forced to properly set some simulation parameters (e.g. the damping factor) to avoid convergence failures. By contrast, the proposed WD method based on the one-dimensional NR solver always converges to the final correct solution.

The superior numerical efficiency and robustness of the

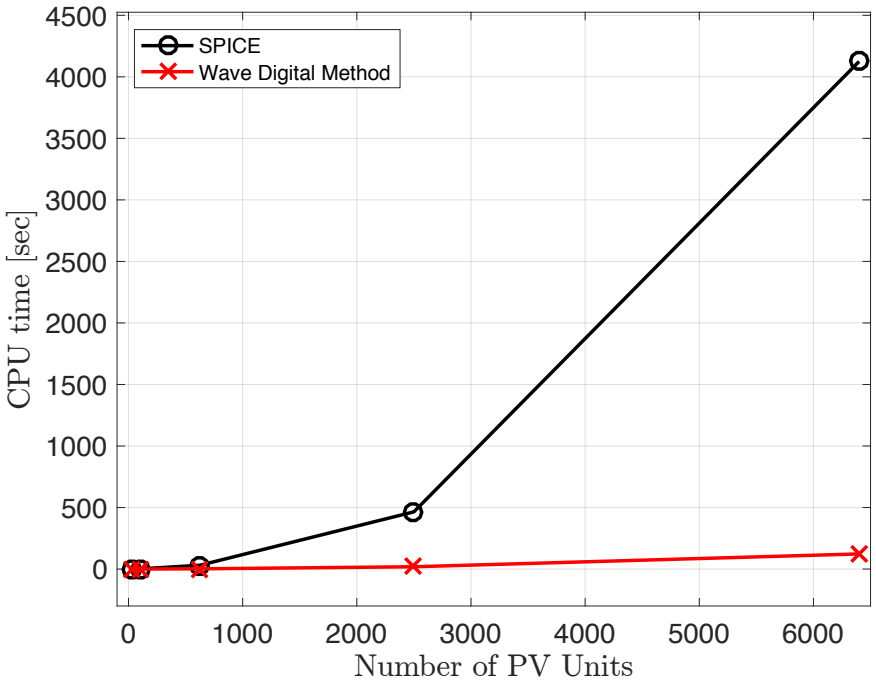

Fig. 19. Efficiency comparison between the proposed Wave Digital method and the MNA-based method. Precise values of the coordinates of the points in this plot are reported in Table II

TABLE II

COMPUTATION TIME FOR PV ARRAYS OF DIFFERENT Sizes

\begin{tabular}{|l|c|c|c|c|c|}
\hline Number of PV Units & 24 & 100 & 625 & 2500 & 6400 \\
\hline $\begin{array}{l}\text { CPU Time MNA-based } \\
\text { Method in seconds }\end{array}$ & 0.68 & 1.8 & 30.75 & 465 & 4125 \\
\hline $\begin{array}{l}\text { CPU Time Wave Digital } \\
\text { Method in seconds }\end{array}$ & 0.1 & 0.25 & 2 & 18.7 & 123 \\
\hline
\end{tabular}

WD method enable possible implementations for the real time control and configuration optimization of large PV plants.

\section{CONClusions AND Future WORK}

In this paper, we have presented an innovative and efficient technique for the modeling and simulation of large networks of nonlinear PV units. The method relies on the usage of Wave Digital (WD) variables and on a scatteringmatrix-based description of the network topology. In this way, the working point of the nonlinear PV units can be found by means of efficient and robust one-dimensional NewtonRaphson (NR) solutions. We have shown how the combination of the proposed scattering matrix description and NR solvers results in an iterative relaxation method that always converges to the PV array solution. More in general, the method proposed in this paper converges for all nonlinear networks that satisfy condition (33). The presented approach is applicable to whichever network topology and handles the critical case where the majority of the elements of the network have a nonlinear characteristic.

The method has been employed to calculate the load characteristics of PV arrays with different topologies under partial shading condition. We have shown how it allows fast exploration of the array power-voltage characteristic with the identification of the Maximum Power Point and of the best array configuration in very short simulation times. The speedup gain compared to standard Spice-like simulation in fact ranges from $7 \times$ for small-size PV arrays to $35 \times$ for large 
arrays made of some thousands elements. This enables real time implementations of the WD method.

Further improvements of the WD algorithm are possible by exploiting the fact that the proposed computational flow is suitable for parallel implementations that can increase even more the already significant numerical efficiency. In addition, the method can also be extended to those cases where the PV units are described by experimental-based models via look-up tables or non-analytical models.

Another possible development would be applying the same approach presented in this manuscript, using alternative definitions of waves [52].

\section{APPENDIX A}

\section{GLOBAL SCATtERING StAGE IMPLEMENTATION DETAILS}

The calculation of 20] in the Global Scattering Stage of the proposed SIM algorithm does not need the explicit formation of the scattering matrix $\mathbf{S}$. In this appendix, we prove this result by referring to the scattering matrix formula (6) based on fundamental cut-set matrix. The dual result can be obtained starting from formula (7) based on fundamental loop matrix.

In fact, by replacing (6) into (20), we derive:

$$
\mathbf{a}^{(k)}=\mathbf{S b}^{(k)}=2 \mathbf{Q}^{T} \mathbf{u}^{(k)}-\mathbf{b}^{(k)}
$$

where $\mathbf{u}^{(k)}=\mathbf{K}_{\mathbf{t}} \mathbf{b}^{(k)}$ is a $t \times 1$ vector.

Hence, multiplying both sides of $(8)$ by $\mathbf{b}^{(k)}$, we find the linear system

$$
\left(\mathbf{Q G Q} \mathbf{Q}^{T}\right) \mathbf{u}^{(k)}=\mathbf{r}^{(k)}
$$

with the right-hand-side vector $\mathbf{r}^{(k)}=\mathbf{Q} \mathbf{G b}^{(k)}$ of size $t \times 1$. The system matrix $\left(\mathbf{Q G Q} \mathbf{Q}^{T}\right)$ is symmetric and positivedefinite and thus it can be efficiently decomposed in the form $\mathbf{L} \mathbf{L}^{T}$ with Cholesky factorization, with $\mathbf{L}$ being a lower triangular matrix. Once factorization is available, at $k$ th iteration of the SIM algorithm and for a given vector $\mathbf{b}^{(k)}$, the evaluation of the associated $\mathbf{a}^{(k)}$ only requires forming the right-hand-side vector $\mathbf{r}^{(k)}$, calculating the vector $\mathbf{u}^{(k)}$ via two backward substitutions and finally evaluating (36). All these calculations only entail vector operations or triangular matrices backsubstitution thus resulting in an almost linear growth of the computational burden as the size of the problem is increased.

\section{APPENDIX B}

\section{BOUNDNESS OF THE SPECTRAL RADIUS}

In this Appendix we provide the proof of Theorem 5.1

Proof Let us start from the property $\mathbf{G S}=\mathbf{S}^{T} \mathbf{G}$, mentioned in Subsection III-A that can be rewritten as $\mathbf{G S Z}=\mathbf{S}^{T}$ or equivalently $\mathbf{G}^{1 / 2} \mathbf{G}^{1 / 2} \mathbf{S} \mathbf{Z}^{1 / 2} \mathbf{Z}^{1 / 2}=\mathbf{S}^{T}$, where $\mathbf{G}=\mathbf{Z}^{-1}$ is the inverse of the diagonal matrix of the free parameters $\mathbf{Z}$. It follows that we can define a matrix $\mathbf{P}$ such that

$$
\mathbf{P}=\mathbf{G}^{1 / 2} \mathbf{S Z}^{1 / 2}=\mathbf{Z}^{1 / 2} \mathbf{S}^{T} \mathbf{G}^{1 / 2}
$$

therefore, $\mathbf{P}$ is symmetric. $\mathbf{P}$ is also involutory, as it can be easily verified that $\mathbf{P P}=\mathbf{I}_{N}$, as $\mathbf{S S}=\mathbf{I}_{N}$ and $\mathbf{G}^{1 / 2} \mathbf{Z}^{1 / 2}=$ $\mathbf{I}_{N}$. It follows that matrix $\mathbf{P}$ is orthogonal.
Let $\lambda$ and $\mathbf{v}$ be an eigenvalue and an eigenvector of matrix $\prod_{k=1}^{K} \mathbf{S D}^{(k)}$ so that we can write

$$
\left(\prod_{k=1}^{K} \mathbf{S D}^{(k)}\right) \mathbf{v}=\lambda \mathbf{v}
$$

Let us then consider another vector $\mathbf{u}$, such that $\mathbf{v}=\mathbf{Z}^{1 / 2} \mathbf{u}$. We can write

$$
\mathbf{G}^{1 / 2}\left(\prod_{k=1}^{K} \mathbf{S D}^{(k)}\right) \mathbf{Z}^{1 / 2} \mathbf{u}=\lambda \mathbf{u}
$$

Exploiting the following two matrix equalities, which can be derived from equation 38,

$$
\mathbf{G}^{1 / 2} \mathbf{S}=\mathbf{P G}^{1 / 2}, \quad \mathbf{S Z}^{1 / 2}=\mathbf{Z}^{1 / 2} \mathbf{P}
$$

and the fact that the diagonal matrices $\mathbf{Z}^{1 / 2}, \mathbf{G}^{1 / 2}, \mathbf{D}^{(k)}$ with $1 \leq k \leq K$, are commuting matrices, 39) can be equivalently written as

$$
\left(\prod_{k=1}^{K} \mathbf{P D}^{(k)}\right) \mathbf{u}=\lambda \mathbf{u}
$$

Assuming $\lambda$ to be the largest eigenvalue of $\prod_{k=1}^{K} \mathbf{S D}^{(k)}$, i.e. $\lambda=\lambda_{\max }$, and applying the 2 -norm operator $\|\cdot\|_{2}$ we have

$$
\left\|\left(\prod_{k=1}^{K} \mathbf{P D}^{(k)}\right) \mathbf{u}\right\|_{2}=\left|\lambda_{\max }\right|\|\mathbf{u}\|_{2}=\rho\left(\prod_{k=1}^{K} \mathbf{S D}^{(k)}\right)\|\mathbf{u}\|_{2}
$$

and, being the 2-norm a submultiplicative norm,

$$
\left\|\left(\prod_{k=1}^{K} \mathbf{P D}^{(k)}\right) \mathbf{u}\right\|_{2} \leq \prod_{k=1}^{K} \rho\left(\mathbf{D}^{(k)}\right)\|\mathbf{u}\|_{2},
$$

as $\left\|\mathbf{D}^{(k)}\right\|_{2}=\rho\left(\mathbf{D}^{(k)}\right)$ for each $k$ and $\|P\|_{2}=1$. Equivalently, as $\left|\lambda_{\max }\right|=\rho\left(\prod_{k=1}^{K} \mathbf{S D}^{(k)}\right)$, we can write

$$
\rho\left(\prod_{k=1}^{K} \mathbf{S D}^{(k)}\right)\|\mathbf{u}\|_{2} \leq \prod_{k=1}^{K} \rho\left(\mathbf{D}^{(k)}\right)\|\mathbf{u}\|_{2},
$$

hence

$$
\rho\left(\prod_{k=1}^{K} \mathbf{S D}^{(k)}\right) \leq \prod_{k=1}^{K} \rho\left(\mathbf{D}^{(k)}\right)
$$

This completes the proof.

\section{REFERENCES}

[1] G. Makrides, B. Zinsser, M. Norton, G. E. Georghiou, M. Schubert, and J. H. Werner, "Potential of photovoltaic systems in countries with high solar irradiation," Renewable and Sustainable Energy Reviews, vol. 14, no. 2, pp. $754-762,2010$.

[2] O. M. Toledo, D. O. Filho, and A. S. A. C. Diniz, "Distributed photovoltaic generation and energy storage systems: A review," Renewable and Sustainable Energy Reviews, vol. 14, no. 1, pp. 506 - 511, 2010.

[3] A. R. Jordehi, "Allocation of distributed generation units in electric power systems: A review," Renewable and Sustainable Energy Reviews, vol. 56, pp. 893 - 905, 2016.

[4] S. Kouro, J. I. Leon, D. Vinnikov, and L. G. Franquelo, "Grid-connected photovoltaic systems: An overview of recent research and emerging pv converter technology," IEEE Industrial Electronics Magazine, vol. 9, no. 1, pp. 47-61, March 2015.

[5] M. E. Meral and F. Diner, "A review of the factors affecting operation and efficiency of photovoltaic based electricity generation systems," Renewable and Sustainable Energy Reviews, vol. 15, no. 5, pp. 2176 - 2184, 2011. 
[6] P. Maffezzoni and D. D'Amore, "Compact electrothermal macromodeling of photovoltaic modules," IEEE Transactions on Circuits and Systems II: Express Briefs, vol. 56, no. 2, pp. 162-166, Feb. 2009.

[7] P. Maffezzoni, L. Codecasa, and D. D'Amore, "Modeling and simulation of a hybrid photovoltaic module equipped with a heat-recovery system," IEEE Transactions on Industrial Electronics, vol. 56, no. 11, pp. 43114318, Nov. 2009.

[8] A. Chatterjee, A. Keyhani, and D. Kapoor, "Identification of photovoltaic source models," IEEE Transactions on Energy Conversion, vol. 26, no. 3, pp. 883-889, Sept. 2011

[9] M. Zagrouba, A. Sellami, M. Bouacha, and M. Ksouri, "Identification of PV solar cells and modules parameters using the genetic algorithms: Application to maximum power extraction," Solar Energy, vol. 84, no. 5, pp. $860-866,2010$.

[10] D. Jena and V. V. Ramana, "Modeling of photovoltaic system for uniform and non-uniform irradiance: A critical review," Renewable and Sustainable Energy Reviews, vol. 52, pp. 400 - 417, 2015.

[11] E. Skoplaki and J. Palyvos, "On the temperature dependence of photovoltaic module electrical performance: A review of efficiency/power correlations," Solar Energy, vol. 83, no. 5, pp. 614 - 624, 2009.

[12] D. L. Manna, V. L. Vigni, E. R. Sanseverino, V. D. Dio, and P. Romano, "Reconfigurable electrical interconnection strategies for photovoltaic arrays: A review," Renewable and Sustainable Energy Reviews, vol. 33 , pp. 412 - 426, 2014.

[13] S. Vemuru, P. Singh, and M. Niamat, "Modeling impact of bypass diodes on photovoltaic cell performance under partial shading," in 2012 IEEE International Conference on Electro/Information Technology, May 2012, pp. $1-5$.

[14] M. L. Orozco-Gutierrez, G. Spagnuolo, J. M. Ramirez-Scarpetta, G. Petrone, and C. A. Ramos-Paja, "Optimized configuration of mismatched photovoltaic arrays," IEEE Journal of Photovoltaics, vol. 6, no. 5, pp. 1210-1220, Sept. 2016.

[15] C. Rodriguez and G. A. J. Amaratunga, "Analytic solution to the photovoltaic maximum power point problem," IEEE Transactions on Circuits and Systems I: Regular Papers, vol. 54, no. 9, pp. 2054-2060, Sept. 2007.

[16] C. Y. Yang, C. Y. Hsieh, F. K. Feng, and K. H. Chen, "Highly efficient analog maximum power point tracking (amppt) in a photovoltaic system," IEEE Transactions on Circuits and Systems I: Regular Papers, vol. 59, no. 7, pp. 1546-1556, July 2012.

[17] Y. Levron and D. Shmilovitz, "Maximum power point tracking employing sliding mode control," IEEE Transactions on Circuits and Systems I: Regular Papers, vol. 60, no. 3, pp. 724-732, March 2013.

[18] M. Uoya and H. Koizumi, "A calculation method of photovoltaic array's operating point for MPPT evaluation based on one-dimensional NewtonRaphson method," IEEE Transactions on Industry Applications, vol. 51, no. 1, pp. 567-575, Jan. 2015.

[19] A. Fettweis, "Wave digital filters: Theory and practice," Proc. of the IEEE, vol. 74, pp. 270-327, Feb. 1986.

[20] S. Bilbao, Wave and Scattering Methods for Numerical Simulation, 1st ed. New York: John Wiley \& Sons, Oct. 2004.

[21] A. Fettweis, "Robust numerical integration using wave-digital concepts," Multidimensional Systems and Signal Processing, vol. 17, no. 1, 2006.

[22] A. Fettweis and K. Meerkötter, "On adaptors for wave digital filters," IEEE Trans. Acoust., Speech, Signal Process., vol. 23, pp. 516-525, Dec. 1975.

[23] D. Fränken, J. Ochs, and K. Ochs, "Generation of wave digital structures for connection networks containing multiport elements," IEEE Trans. Circuits and Systems I: Regular Papers, vol. 52, pp. 586-596, March 2005.

[24] K. J. Werner, J. O. Smith, and J. S. Abel, "Wave digital filters adaptors for arbitrary topologies and multiport linear elements," in Proc. 18th Conf. Digital Audio Effects, Trondheim, Norway, Nov. 30 - Dec. 32015 , pp. 379-386.

[25] K. J. Werner, W. R. Dunkel, M. Rest, M. J. Olsen, and J. O. Smith, "Wave digital filter modeling of circuits with operational amplifiers," in 2016 24th European Signal Processing Conference (EUSIPCO), Budapest, Hungary, Aug. 2016, pp. 1033-1037.

[26] M. Verasani, A. Bernardini, and A. Sarti, "Modeling Sallen-Key audio filters in the wave digital domain," in Proc. 42nd Int. Conf. on Acoustics, Speech and Signal Process. (ICASSP), March 5-9 2017, pp. 431-435.

[27] K. Meerkötter and R. Scholz, "Digital simulation of nonlinear circuits by wave digital filter principles," in IEEE Int. Symp. Circuits Syst., vol. 1, June 1989, pp. 720-723.

[28] T. Felderhoff, "A new wave description for nonlinear elements," in 1996 IEEE Int. Symposium on Circuits and Systems. ISCAS 96, vol. 3, May 1996, pp. 221-224.
[29] A. Sarti and G. De Poli, "Toward nonlinear wave digital filters," IEEE Trans. Signal Process., vol. 47, pp. 1654-1668, June 1999.

[30] A. Sarti and G. De Sanctis, "Systematic methods for the implementation of nonlinear wave-digital structures," IEEE Trans. Circuits Syst. I, Reg. Papers, vol. 56, pp. 460-472, Feb. 2009.

[31] G. De Sanctis and A. Sarti, "Virtual analog modeling in the wave-digital domain," IEEE Trans. Audio, Speech, Language Process., vol. 18, pp. 715-727, May 2010.

[32] A. Bernardini, K. J. Werner, A. Sarti, and J. O. Smith, "Modeling nonlinear wave digital elements using the Lambert function," IEEE Trans. Circuits and Systems I: Regular Papers, vol. 63, pp. 1231-1242, August 2016

[33] A. Bernardini, K. J. Werner, A. Sarti, and J. O. Smith, "Multi-port nonlinearities in wave digital structures," in Proc. IEEE Int. Symp. Signals Circuits Syst., Iasi, Romania, July 9-10 2015.

[34] A. Bernardini, K. J. Werner, A. Sarti, and J. O. Smith, "Modeling a class of multi-port nonlinearities in wave digital structures," in Proc. European Signal Process. Conf. (EUSIPCO), Nice, France, Aug. 31 Sept. 4 2015, pp. 669-673.

[35] A. Bernardini and A. Sarti, "Dynamic adaptation of instantaneous nonlinear bipoles in wave digital networks," in Proc. 2016 24th European Signal Processing Conference (EUSIPCO), Budapest, Hungary, Aug. 2016, pp. $1038-1042$

[36] S. D'Angelo, J. Pakarinen, and V. Välimäki, "New family of wavedigital triode models," IEEE Trans. Audio, Speech, Language Process. vol. 21, pp. 313-321, Feb. 2013.

[37] S. Petrausch and R. Rabenstein, "Wave digital filters with multiple nonlinearities," in Proc. European Signal Process. Conf. (EUSIPCO), vol. 12, Vienna, Austria, Sept. 2004

[38] T. Schwerdtfeger and A. Kummert, "Newton's method for modularitypreserving multidimensional wave digital filters," in IEEE 9th Int Workshop on Multidimensional ( $n D)$ Systems ( $n D S)$, Vila Real, Portugal, Sept. 7-9 2015, pp. 1-6.

[39] K. J. Werner, V. Nangia, J. O. Smith, and J. S. Abel, "Resolving wave digital filters with multiple/multiport nonlinearities," in Proc. 18th Conf. Digital Audio Effects, Trondheim, Norway, Nov. 30 - Dec. 3 2015, pp. 387-394.

[40] L. Lombardi, P. Belforte, and G. Antonini, "Digital wave simulation of quasi-static partial element equivalent circuit method," IEEE Transactions on Electromagnetic Compatibility, vol. 59, no. 2, pp. 429-438, April 2017.

[41] S. Seshu and M. B. Reed, Linear graphs and electrical networks, 1st ed. AddisonWesley Publishing Company, Inc., Reading, MA, 1961.

[42] L. Chua, C. Desoer, and E. Kuh, Linear and Nonlinear Circuits, ser. Circuits and systems. McGraw-Hill, 1987.

[43] G. O. Martens and K. Meerkötter, "On n-port adaptors for wave digital filters with application to a bridged-tee filter," in Proc IEEE Int. Symp. Circuits Syst., Munich, Germany, Apr. 1976, p. 514517.

[44] G. O. Martens and H. Le, "Wave digital adapters for reciprocal secondorder sections," IEEE Transactions on Circuits and Systems, vol. 25, no. 12, pp. 1077-1083, Dec. 1978.

[45] K. Meerkötter and D. Franken, "Digital realization of connection networks by voltage-wave 2-port adapters," AEU - International Journal of Electronics and Communications, vol. 50(6), pp. 362-367, 1996.

[46] M. G. Villalva, J. R. Gazoli, and E. R. Filho, "Comprehensive approach to modeling and simulation of photovoltaic arrays," IEEE Transactions on Power Electronics, vol. 24, no. 5, pp. 1198-1208, May 2009.

[47] K. Ishaque, Z. Salam, and H. Taheri, "Simple, fast and accurate twodiode model for photovoltaic modules," Solar Energy Materials and Solar Cells, vol. 95, no. 2, pp. 586 - 594, 2011.

[48] A. Ortiz-Conde, D. Lugo-Munoz, and F. J. Garcia-Sanchez, "An explicit multiexponential model as an alternative to traditional solar cell models with series and shunt resistances," IEEE Journal of Photovoltaics, vol. 2, no. 3, pp. 261-268, July 2012.

[49] K. J. Werner, V. Nangia, A. Bernardini, J. O. Smith, and A. Sarti, "An improved and generalized diode clipper model for wave digital filters," in Proc. Conv. Audio Eng. Soc., New York, NY, Oct. 29 - Nov. 12015.

[50] A. Bernardini and A. Sarti, "Canonical piecewise-linear representation of curves in the wave digital domain," in Proc. European Signal Process. Conf. (EUSIPCO), Kos island, Greece, Aug. 2017, pp. 1165-1169.

[51] Virtuoso Spectre Circuit Simulator RF Analysis User Guide, Cadence Design Syst., San Jose CA, Product Version 7.2 ed., May 2010.

[52] A. Bernardini and A. Sarti, "Biparametric wave digital filters," IEEE Transactions on Circuits and Systems I: Regular Papers, vol. 64, no. 7. pp. 1826-1838, July 2017. 


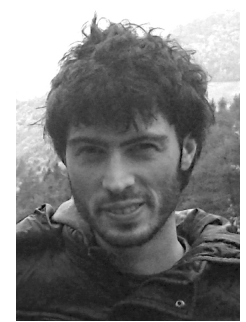

Alberto Bernardini ( $\left.\mathrm{S}^{\prime} 16\right)$ received his B.S. from the University of Bologna, Italy, in 2012; and his M.S. degree (cum laude) from the Politecnico di Milano, Italy, in 2015, both in Computer Engineering. He is currently a Ph.D. candidate in Information Engineering at the Dipartimento di Elettronica, Informazione and Bioingegneria of the Politecnico di Milano, Italy. His main research interests are audio signal processing and modeling of nonlinear systems.

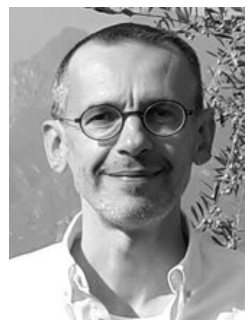

Paolo Maffezzoni (M'08-SM'15) received the Laurea degree (summa cum laude) in electrical engineering from Politecnico di Milano, Italy, in 1991 and the $\mathrm{Ph} . \mathrm{D}$. degree in electronic instrumentation from Universita di Brescia, Italy, in 1996. Since 1998, he has been with Politecnico di Milano, where he is a Professor of Electrical Engineering. His current research interests include modeling and simulation of oscillatory devices for engineering applications, synchronization phenomena, nonlinear circuits and systems, analog and mixed-signal electronics, stochastic simulation, unconventional computing. He has over 130 research publications. $\mathrm{He}$ currently serves as an Associate Editor for the IEEE TRANSACTIONS ON CIRCUITS AND SYSTEMS-PART I: REGULAR PAPERS. He served as an Associate Editor for IEEE TRANSACTIONS ON COMPUTER-AIDED DESIGN OF INTEGRATED CIRCUITS AND SYSTEMS and as a member of the Technical Program Committee for the IEEE/ACM Design Automation Conference.

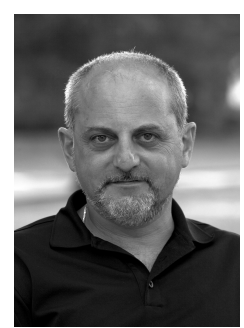

Augusto Sarti (M'04-SM'13) received his M.S. in electronic engineering from the University of Padua, Italy, in 1988. He then received his Ph.D. in Information Engineering in 1993, again from the University of Padua, Italy. His graduate studies were in a joint graduate program with the University of California, Berkeley. In 1993, he joined the faculty of the Politecnico di Milano, Italy. In 2013, he also joined the University of California, Davis, as an Adjunct Professor. His research interests are in the area of multimedia signal processing, with particular focus on sound analysis, synthesis and processing; space-time audio processing; geometrical acoustics; and music information extraction. He has also worked on problems of image analysis and 3D vision. He coauthored over 250 scientific publications on international journals and congresses as well as numerous patents in the multimedia signal processing area. $\mathrm{He}$ coordinates the activities of the Musical Acoustics Lab and of the Sound and Music Computing Lab of the Politecnico di Milano. He has been the promoter/coordinator and/or contributor to numerous (20+) European projects. $\mathrm{He}$ is an active member of the IEEE Technical Committee on Audio and Acoustics Signal Processing, and is in the Editorial Board of the IEEE.
Luca Daniel (S'98-M'03) received the Ph.D. de-

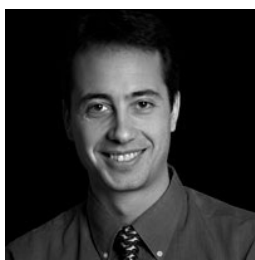

gree in electrical engineering from the University of California, Berkeley, CA, USA, in 2003. He is currently a Full Professor in the Electrical Engineering and Computer Science Department of the Massachusetts Institute of Technology, Cambridge, MA, USA (MIT). Industry experiences include HP Research Labs, Palo Alto, CA, USA (1998) and Cadence Berkeley Labs, CA, USA (2001). His current research interests include integral equation solvers, uncertainty quantification and parameterized model order reduction, applied to RF circuits, silicon photonics, MEMs, magnetic resonance imaging scanners, and the human cardiovascular system. Prof. Daniel was the recipient of the 1999 IEEE TRANSACTIONS ON POWER ELECTRONICS best paper award; the 2003 best Ph.D. thesis awards from the Electrical Engineering and the Applied Math departments at UC Berkeley; the 2003 ACM Outstanding Ph.D. Dissertation Award in Electronic Design Automation; the 2009 IBM Corporation Faculty Award; the 2010 IEEE Early Career Award in Electronic Design Automation; the 2014 IEEE TRANSACTIONS ON COMPUTER-AIDED DESIGN OF INTEGRATED CIRCUITS AND SYSTEMS best paper award; and ten awards in conferences. Prof Daniel recently received the 2016 Teaching Award from the MIT School of Engineering. 